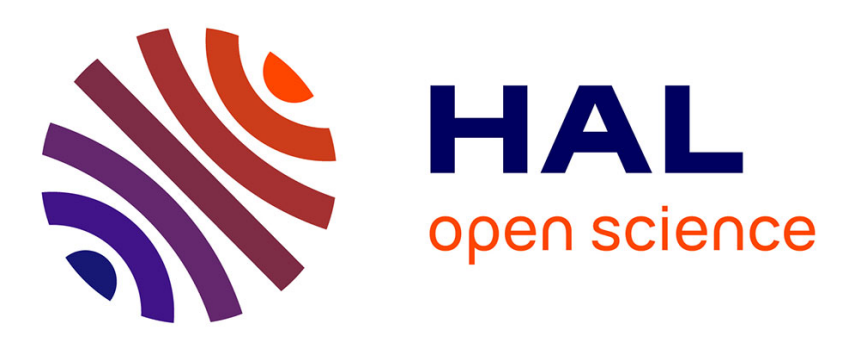

\title{
Periodic forced flow in a nanosecond pulsed cold atmospheric pressure argon plasma jet
}

Thibault Darny, Gérard Bauville, Michel Fleury, Stéphane Pasquiers, João

Santos Sousa

\section{- To cite this version:}

Thibault Darny, Gérard Bauville, Michel Fleury, Stéphane Pasquiers, João Santos Sousa. Periodic forced flow in a nanosecond pulsed cold atmospheric pressure argon plasma jet. Plasma Sources Science and Technology, 2021, 30, 105021 (19pp). 10.1088/1361-6595/ac2a18 . hal-03364904

\section{HAL Id: hal-03364904 https://hal.science/hal-03364904}

Submitted on 5 Oct 2021

HAL is a multi-disciplinary open access archive for the deposit and dissemination of scientific research documents, whether they are published or not. The documents may come from teaching and research institutions in France or abroad, or from public or private research centers.
L'archive ouverte pluridisciplinaire HAL, est destinée au dépôt et à la diffusion de documents scientifiques de niveau recherche, publiés ou non, émanant des établissements d'enseignement et de recherche français ou étrangers, des laboratoires publics ou privés. 
ACCEPTED MANUSCRIPT

\section{Periodic forced flow in a nanosecond pulsed cold atmospheric pressure argon plasma jet}

To cite this article before publication: Thibault Darny et al 2021 Plasma Sources Sci. Technol. in press https://doi.org/10.1088/1361-6595/ac2a18

\section{Manuscript version: Accepted Manuscript}

Accepted Manuscript is "the version of the article accepted for publication including all changes made as a result of the peer review process, and which may also include the addition to the article by IOP Publishing of a header, an article ID, a cover sheet and/or an 'Accepted

Manuscript' watermark, but excluding any other editing, typesetting or other changes made by IOP Publishing and/or its licensors"

This Accepted Manuscript is @ 2021 IOP Publishing Ltd.

During the embargo period (the 12 month period from the publication of the Version of Record of this article), the Accepted Manuscript is fully protected by copyright and cannot be reused or reposted elsewhere.

As the Version of Record of this article is going to be / has been published on a subscription basis, this Accepted Manuscript is available for reuse under a CC BY-NC-ND 3.0 licence after the 12 month embargo period.

After the embargo period, everyone is permitted to use copy and redistribute this article for non-commercial purposes only, provided that they adhere to all the terms of the licence https://creativecommons.org/licences/by-nc-nd/3.0

Although reasonable endeavours have been taken to obtain all necessary permissions from third parties to include their copyrighted content within this article, their full citation and copyright line may not be present in this Accepted Manuscript version. Before using any content from this article, please refer to the Version of Record on IOPscience once published for full citation and copyright details, as permissions will likely be required. All third party content is fully copyright protected, unless specifically stated otherwise in the figure caption in the Version of Record.

View the article online for updates and enhancements. 


\title{
Periodic forced flow in a nanosecond pulsed cold atmospheric pressure argon plasma jet
}

\author{
Thibault DARNY, Gérard BAUVILLE, Michel FLEURY, Stéphane PASQUIERS and João SANTOS SOUSA
}

Université Paris-Saclay, CNRS, Laboratoire de Physique des Gaz et des Plasmas, 91405 Orsay, France

\section{Abstract}

This paper is devoted to the study of the argon flow modification in a cold atmospheric pressure plasma jet driven by nanosecond high voltage (HV) pulses, from single to multiple HV shots applications. A schlieren optical bench has been designed in order to visualize the argon flow downstream expansion in quiescent air, for moderate flow rates below 1 standard liter per minute. A coupled approach is used between charge coupled device (CCD) schlieren imaging and intensified CCD (ICCD) plasma plume imaging, both time-resolved. It is shown that the application of only one HV pulse (i.e. single HV shot) is enough to disturb the flow. The disturbed flow exhibits ripple propagation, on a timescale similar to the flow velocity. When operating in double HV shots, the second ionization wave can be used as a probe, to instantly visualize the flow structure any time after the first HV pulse application. For some flow rates, the ripple can increase in amplitude up to the point when it strongly deforms, or even stops, the plasma plume expansion, after which it is entrained by the flow and the plasma plume retrieves its full usual expansion. When a series of HV pulses are applied, the maximal disturbance of the flow is achieved for a certain pulse repetition frequency (PRF), specific of each flow rate. It is associated with ripples alternation in the plasma plume, in a 3D helicallike arrangement. For greater PRF, the ripples progressively vanish, and the flow is clearly less disturbed. Once the ripples have vanished, increasing further the PRF does not change the plasma plume and flow structures. We suggest that the repetitive plasma ignition mechanically forces the flow inside the capillary with consequences on the global flow structure, similarly to a forced backward-facing step flow with actuator.

\section{Introduction}

Cold atmospheric pressure plasma jets (CAPPJ) have been extensively studied and used both because of their flexibility of uses and their ability to produce a wide range of reactive species of interest. These plasma devices allow to target a large panel of applications, in biomedicine [1]-[7], decontamination and depollution [8]-[10], analytical chemistry [11]-[13] and material processing [14], [15]. Also, CAPPJ are challenging for experimental and numerical fundamental studies, because of the need of highly time- and space-resolved non-intrusive characterization 
techniques [16]-[23] and the coupling of phenomena resulting from different timescale features covering several orders of magnitude [24]. To this regard, a lot of effort has been made to study the interaction between the plasma and the gas flow. As the production of the reactive species occurs in the plasma plume and it is sensitive to the mixing with the ambient air, one crucial mechanism for a better control of the reactive species production is the plasma-flow interaction. Since the first study on plasma-flow interaction in CAPPJ by Oh et al. [25], a lot of other studies have been devoted to this aspect [26]-[49]. Schlieren imaging is a convenient method for flow visualization in CAPPJ [50].

A general ascertainment is that, in terms of flow modification, there are more studies on helium CAPPJ [26]-[34], [38], [43]-[49] than on argon CAPPJ [35]-[37], [39]-[42]. One possible reason could be due to the simple fact that an argon jet expanding in air is more difficult to visualize than a helium jet, when using schlieren imaging, because of the much smaller difference of density between argon and ambient air. In the context of applications, the use of argon CAPPJ instead of helium CAPPJ might be advantageous in some cases. This is not only because argon is cheaper than helium, but also because argon metastables have almost half the energy of helium metastables, allowing for more delicate surface treatments [11]$[13],[16]$. Concerning the studies on the flow modification with argon CAPPJ, it should be pointed out that, except for the study of Shao et al. [40] on an AC powered argon CAPPJ, all the other studies considered argon CAPPJ powered in RF [35], [36], [41], [42] and microwave [37], [39]. For these latter power schemes, significant gas heating is necessarily expected. In those studies, it has been identified that large-scale coherent vortex structures are present in the argon shear layer. Those vortex structures have been clearly imaged in the study of Zhang et al. [36]. According to the authors, the plasma locally heats the argon, which results in a decrease of the gas density, an increase of the gas velocity and, thus, in a vortex formation in the unstable shear layer that reduces the length of the argon potential core. Kubečka et al. [39] have also found that the perturbation is ignited in the plasma plume region and is released when the plasma power falls down. This causes a sudden drop in the pressure inside the capillary and the generation of a back-travelling flow. The authors have also shown that this perturbation is entrained in the argon flow, at the flow velocity. With an AC powered argon CAPPJ, Shao et al. [40] have notably reported that forward momentum could be given to the flow, with a preservation of the laminar flow structure. The jet was oriented horizontally, and a low flow rate $(0.2 \mathrm{~s} / \mathrm{m})$ was used. With a numerical simulation, they have concluded that an electrohydrodynamic (EHD) effect explains their observation. For helium CAPPJ, the studies that evidenced an EHD effect with ionic wind momentum transfer were generally made at low Re with generally DC pulsed CAPPJ [28], [38], [45], [48], but not systematically [30], [34]. Concerning the origin of the laminar to turbulent transition observed with plasma generation in helium CAPPJ [25], [29], [31], [32], Hasan et al. [48] have shown that a significant increase of the Reynolds number $(R e)$ is an unlikely explanation. To explain the onset of turbulence at low Re, Whalley et al. [27] have emphasized on the role of small perturbations in the highly unstable helium shear layer, which grow as they move downstream, based on the literature of free round jets instabilities [51], [52]. The authors have shown that the gas temperature increase and the EHD force due to the plasma have a negligible effect on the flow velocity field. Using particle image velocimetry, Morabit et al. [26] 
have demonstrated that the plasma generation only results in a minor increase of the gas velocity at the capillary outlet. Furthermore, by measuring an increase of the quenching rate of the laser induced fluorescence of $\mathrm{OH}$, not only in the turbulent region but at all spatial positions within the helium jet, they have concluded that the plasma generation plays a major role on the development of the small instabilities on the highly unstable shear layer. The origin of the perturbation generated by the ionization wave (IW) ignition was given by Lietz et al. [44]. With a numerical modelling of helium CAPPJ, the authors have shown that the IW ignition at the inner electrode, inside the capillary, results in a fast gas heating that doubles the pressure in the boundary layer. Then, the highly unstable helium shear layer, downstream of the capillary outlet, is perturbed by the resulting acoustic wave propagation. According to the model, the application of only one voltage pulse (single HV shot) is enough to destabilize the helium shear layer. They have consequently demonstrated that the flow modification does not start in the plasma plume, but rather inside the capillary in the vicinity of the inner electrode. Hence, the excitation of the unstable helium shear layer does not depend neither on the gas heating nor on the EHD force in the plasma plume region. Van Doremael et al. [43] have precisely quantified the effect of the gas heating and the EHD force in the plasma plume. The rotational spectra of $\mathrm{OH}$ and $\mathrm{N}_{2}$ were used for the measurement of the gas temperature, while Rayleigh scattering was used for the determination of the air distribution in the helium flow. The authors have demonstrated that the flow modification was neither due to plasma heating nor to the EHD force related to the ionization wave (IIW) propagation in the plasma plume. It should be pointed out that in [27], [26] and [43], CAPPJ are not similarly powered. While in [27] and [26] AC at respectively $50 \mathrm{kHz}$ and $20 \mathrm{kHz}$ was used, in [43] DC pulsed at 5 $\mathrm{kHz}$ was used. The flow perturbation resulting from plasma generation and its entrainment by the helium flow was first evidenced by Boselli et al. [29] and, then, by Qaisrani et al. [46]. Boselli et al. [29] are also most likely the first to have suggested the effect of a pressure variation to explain the phenomena. In the study of Boselli et al. [29], the CAPPJ was pulsed powered (with damped voltage oscillations). For the first voltage period with the highest amplitude, the risetime is of about $25 \mathrm{~ns}$, and subsequent voltage oscillations are damped in 500 ns. Qaisrani et al. [46] have used DC pulsed CAPPJ with a pulse duration of $1 \mu \mathrm{s}$, but the waveform was not shown and the risetime not mentioned. In the model of Lietz et al. [44], a single voltage pulse of 50 ns duration and 30 ns risetime was considered. It could then be expected that a DC pulsed plasma jet with ns and $\mu$ s pulse duration allows to trigger this flow perturbation. Nevertheless, to the extent that the pressure variation relies on the IW ignition, it cannot be excluded that the same phenomenon may occur with an AC excitation. However, given that it is not possible to precisely control when the IW is ignited when using an AC power, this is potentially more difficult to be confirmed. With a helium CAPPJ similar to that of Qaisrani et al. [46], powered by DC square pulses of $10 \mu$ s duration, Xian et al. [47] have found that one precise pulse repetition frequency (PRF) can maximize the flow perturbation $(6 \mathrm{kHz}$ in their case). They have also observed twisted plasma plume shapes in a specific PRF range. Outside this range, straight plasma plumes were observed. The authors have also suspected the role played by pressure variations in their observations. Similar observations with a flow rate-PRF dependence of the plasma plume shape, in a Neon CAPPJ, were previously reported in [53]. Up to now, regarding the transition from a structured laminar flow to a more disturbed flow, the role of the plasma perturbations on the shear layer excitation, resulting in the 
appearance and growth of vortex structures, has been well evidenced, both for helium and argon CAPPJ. Nevertheless, it is worth mentioning that the origin and the magnitude of the perturbations due to argon plasma jets powered in RF or microwave and helium plasma jets powered with DC pulses or in AC differ widely. Using argon CAPPJ powered in RF or microwave automatically implies a consequent gas heating by the plasma. On the other hand, in helium CAPPJ powered with DC pulses and in AC, the magnitude of the small perturbations resulting from the plasma ignition is enough to destabilize the highly unstable helium shear layer. It is worth mentioning that according to fluid mechanics' studies on free round jets of variabledensity, there is a fundamental distinction to highlight between helium and argon jets expanding in quiescent air. Kyle and Sreenivasan [54] have indeed found that when the density ratio between the jet fluid at the nozzle and the fluid present in the surroundings is greater than 0.6 , shear layer instabilities comparable to those of a constant density jet occur. But, for a density ratio lower than 0.6 , they have reported that intense oscillation instabilities occur in the shear layer. This mode, called by the authors "oscillating mode", is characterized by pulsation frequencies, with the onset depending on the nozzle diameter, the gas velocity at the nozzle exit, and the density ratio between the jet and its surroundings [55]. Comparing helium and argon jets expanding in quiescent air, at atmospheric pressure and ambient temperature, the density ratio changes by one order of magnitude, from 0.13 to 1.32 , respectively. This observation is of importance because, as an universal trend, the literature on variable-density jets demonstrates that the intense shear layer instabilities in the oscillating mode with associated formation of large-scale coherent vortex structures and periodic features cannot occur for density ratios greater than 0.6 [54]-[59]. Then, it can be expected that a helium shear layer is more easily perturbated by small magnitude perturbations, compared to an argon shear layer, because the latter is fundamentally less unstable. However, a strong local heating of the gas, such as with argon CAPPJ powered in RF or microwave, can likely induce variations of density ratio in the heated gas region in such manner that the strong oscillation mode in the shear layer can be triggered, similarly to pool fires [55].

Also, it is worth mentioning that among the wide variety of CAPPJ devices found in the literature, independently of the buffer gas used, the gas injection geometry, resulting from the HV electrode assembly, widely differs. Depending on the gas injection geometry used, the gas can flow, prior to its expansion outside of the capillary, through a hollowed inner HV electrode inserted inside the capillary [12],[13],[17],[16], [19], $[20],[28],[44],[45],[53],[60],[61]$ or around a needle HV electrode [26],[29],[38],[43],[46],[47]. External HV ring electrodes wrapped around the capillary are also used [25],[27],[49],[32][34] and, in this case, do not interfere with the gas flow. Especially with a hollowed inner HV electrode inserted inside the/capillary, the gas flowing from the electrode to the capillary undergoes a sudden expansion at their junction. This kind of geometry has been extensively studied to model flow separation in fluid mechanics and it is known as the Backward-Facing Step (BFS). A recent review of BFS flow mechanisms has been published by Chen et al. [62], with exhaustive descriptions of experimental and numerical studies and also of flow control strategies. Briefly, BFS flows are essentially characterized by the development of highly unstable and unsteady flow regions, strongly coupled between each other. The vortex dynamic between the different coupled regions can impact the whole flow structure. This 
problem is often considered as a difficult test case for computational fluid dynamics, because it approaches the complexity degree of a real flow. Various Re have been investigated, for air and water flows: low (laminar regime), moderate (transitional regime) and high (turbulent regime). Depending on the geometric conditions and on the Re [63], very complex series of vortices can be generated and a large variety of periodic modes can be defined. Frequency bands rather than frequency peaks are usually observed because of a complex retroaction mechanism through back traveling pressure waves [64]. Self-sustained oscillation modes have also been observed and analyzed [65], due to the absolutely unstable flow. Some remarkable coherent structures have been reported: hairpin-like structure [66], spanwise spiraling flows [67] and stationary helical vortices (SHV) with double helical structures wrapped around the vortex tube [68].

Insofar as the shear layer of a free round jet is already fundamentally unstable, with a variable degree depending on the density ratio between the fluid jet and the surroundings, attention must be paid to consider all other potential sources of flow instabilities, especially the gas injection geometry resulting from the HV electrode assembly used. To our knowledge, this aspect has not yet been considered in the field of CAPPJ. It should also be noticed that the plasma effect on the flow is very often described as "flow modifications" or "flow perturbations". It contrasts with the term "flow control" usually used in the field of surface DBD plasma actuators, where it has been shown that the flow structure can be changed and controlled under appropriate plasma actuation parameters [69]-[71]. In this paper, we have experimentally studied the plasma-flow interaction in a cold atmospheric pressure argon plasma jet driven by nanosecond high voltage (HV) pulses, with an inner hollowed electrode (hollowed needle) inserted inside the capillary. The effect of the application of a single HV pulse (single HV shot) and of series of HV pulses at several PRF (multiple HV shots) are studied for a flow rate range between 400 and 800 standard cubic centimeters per minute $(\mathrm{sccm})$. This flow rate range allows to work with a plasma plume expansion occurring in a laminar flow region. Both the argon flow and the plasma plumes emission are visualized with time-resolved imaging. Details of the experimental setup are given in section 2: the plasma jet reactor geometry, the electrical parameters, the triggering system for temporal visualization and the specificities of the schlieren optical bench used. Results and discussions are presented in section 3: argon flow structure and plasma plume emission modification for single, double, and a series of multiple shots of HV pulses at several PRF; analogies with flow control actuation reported in BFS geometry are developed. Concluding remarks and perspectives are given in section 4.

\section{Experimental setup}

The geometry of the plasma jet reactor used is shown in Figure 1(a.). This DBD coaxial geometry is the same as that used in [12], [13], [16]. A cylindrical hollowed stainless-steel needle (HV electrode; $1.2 \mathrm{~mm}$ inner diameter, $1.7 \mathrm{~mm}$ outer diameter and $100 \mathrm{~mm}$ long) was inserted inside the capillary, a cylindrical dielectric quartz tube of $1.7 \mathrm{~mm}$ inner diameter and $4.3 \mathrm{~mm}$ outer diameter. The distance between the HV electrode outlet and the capillary outlet is $10 \mathrm{~mm}$. Argon (ALPHAGAZ 1 purity, Air Liquid) was flushed through the HV electrode, then 
expanding for $10 \mathrm{~mm}$ through the quartz capillary and finally downstream of the capillary outlet into quiescent air. The plasma jet reactor was oriented vertically, from top to bottom, in the downstream direction. A coper foil ( $10 \mathrm{~mm}$ wide and $100 \mu \mathrm{m}$ thick) was wrapped around the quartz capillary tube and grounded. Its center is aligned with the HV electrode outlet. The argon flow rate was adjusted between 300 and $800 \mathrm{sccm}$, using a mass flow controller (Brooks Instruments SLA 5850S). The full scale of the flow controller is $1100 \mathrm{sccm}$. According to the datasheet, the precision of the flow rate values is $0.7 \%$ of the full scale for the flow rate range used, which corresponds to $8 \mathrm{sccm}$.

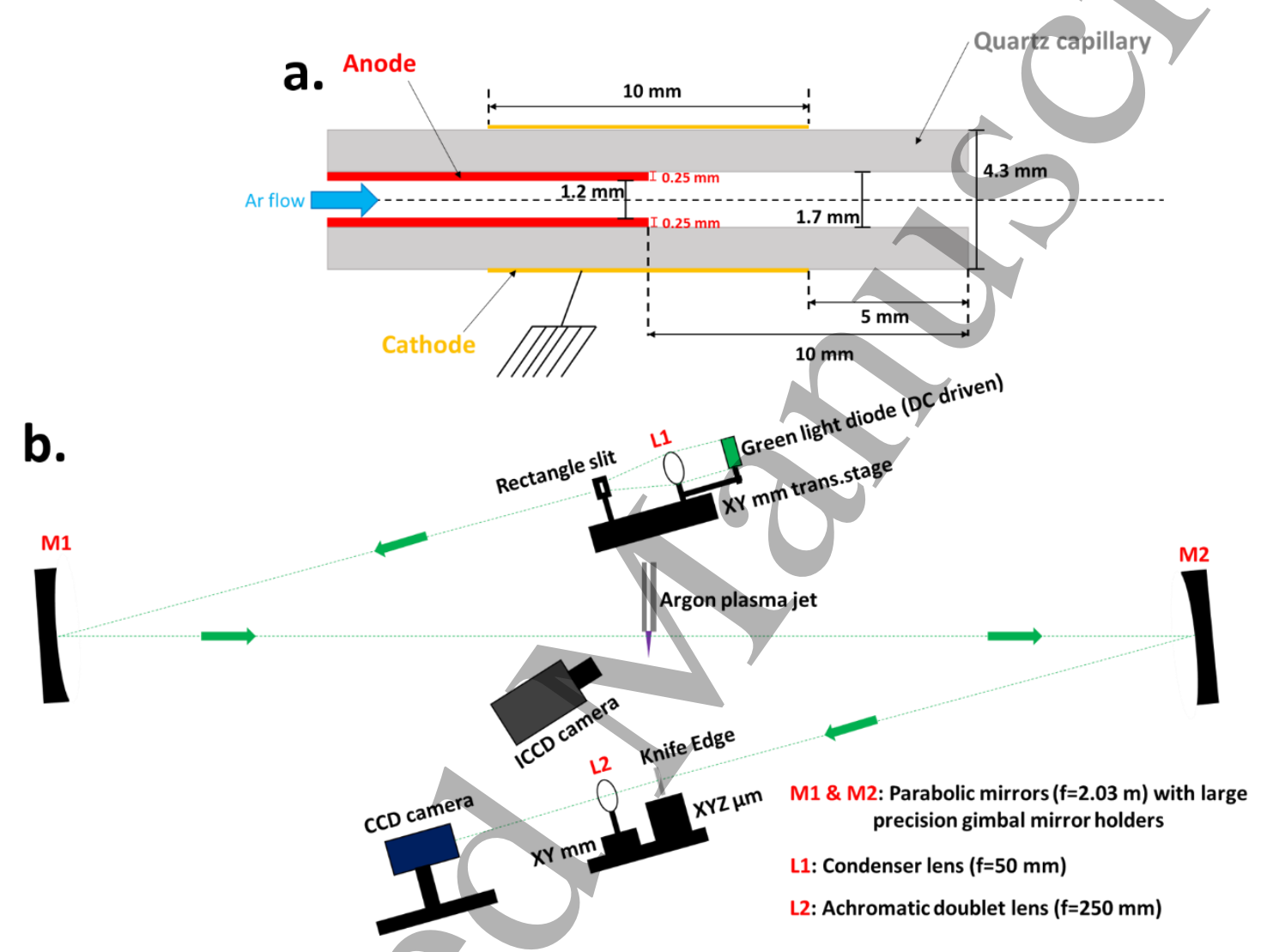

Figure $1: 2 D$ section of the cylindrical geometry of the argon plasma jet reactor used (a.). Schlieren optical bench with the argon plasma jet oriented in the downstream direction (b.).

To visualize the argon flow expansion into quiescent air, a schlieren optical bench was designed (Z-type configuration), as shown in Figure 1(b.). Two parabolic mirrors of $2.03 \mathrm{~m}$ focal length and $203 \mathrm{~mm}$ diameter (Edmund Optics ${ }^{\circ}$ ) were set in large precision gimbal mirror holders (Optosigma ), allowing horizontal and vertical tilt control. The two mirrors are distant of $4.25 \mathrm{~m}$. A green light diode, driven by a DC generator, was used as a light source. The light was first focused through a condenser lens to an adjustable rectangle slit (aligned with the highest dimension in the vertical direction). The whole illumination assembly was set on a XY millimeter translation stage and the slit was positioned at the focal length of the first mirror. The knife edge (razor blade) was set at the focal point of the second mirror using a XYZ micrometrics translation stage for fine adjustments of the knife position and of the cutoff degree. The schlieren image was formed on the charge coupled device (CCD) camera sensor (Retiga $6^{\circ}, 2288 \times 2200$ pixels) using an achromatic doublet lens ( $250 \mathrm{~mm}$ focal length), moved 
with a 2-axis millimeter translation stage between the knife edge and the camera. In our experimental conditions, a cutoff of about $10 \%$ is still high enough to resolve the argon flow in air. According to Seetles [72], the sensitivity is about few arcseconds when the "hand test " is validated, which is the case with the present schlieren optical bench. The resolution on the images recorded by the CCD camera is $0.038 \mathrm{~mm} / \mathrm{pxl}$, with an error of $2 \%$. When the position of the region of interest was measured downstream of the capillary outlet (appearing in black on the top of each schlieren image), the error of the delimited position was assumed to be 5 pixels ( $200 \mu \mathrm{m})$.

In what concerns the temporal resolution, the CDD camera used for schlieren imaging was operated in trigged mode, as shown in Figure 2(a.).
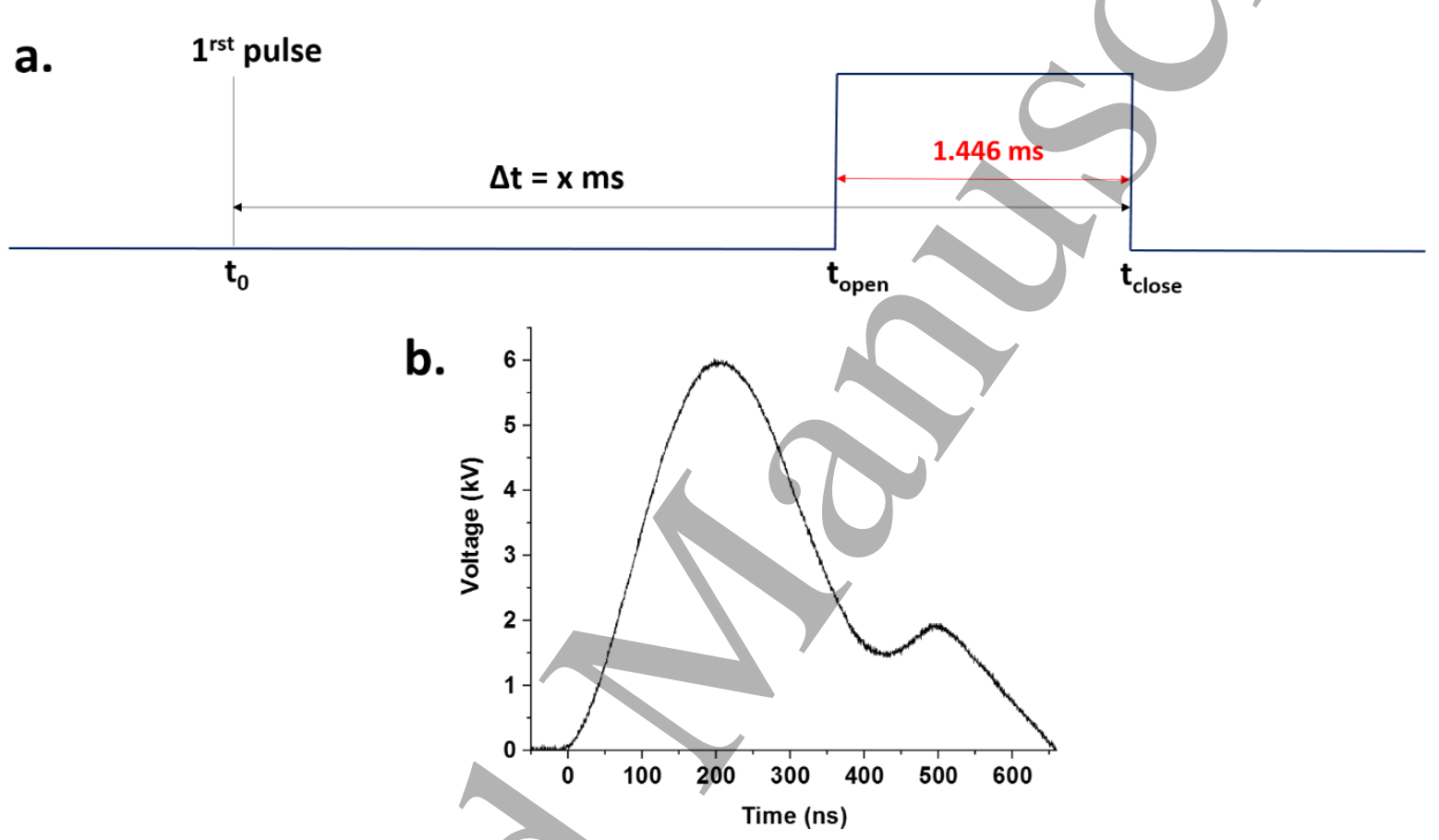

Figure 2 : Gate mode of the CCD camera used for time-resolved schlieren imaging (a.). Temporal evolution of the HV pulse applied on the anode (b.).

A delay generator (Standford DG 535 ) was used to gate the CCD camera, with the control of the acquisition duration and delay. We have measured that the minimum integration time (for a linear captor response with exposure time) is $1.446 \mathrm{~ms}$. This integration time value was used for the acquisition of all the schlieren images shown in this paper. The delay generator also trigs another one (Standford DG $635^{\circ}$ ), which drives the HV pulse sequence in burst mode. The temporal evolution of the HV pulse is shown in Figure 2(b.). For all the conditions shown in the present paper, the applied voltage amplitude is fixed at $6 \mathrm{kV}$, for which the discharge peak current is of about $175 \mathrm{~mA}$ and the energy deposited per pulse is of about $20 \mu \mathrm{J}$ [11],[13], $[16],[20]$. For each burst event, the delay of the first HV pulse, the number of HV pulses and the PRF can be controlled. By varying the delay between the HV pulse in single shot (or the first HV pulse of the series if multiple shots are applied) and the CCD camera gate, it is possible to follow the time dynamics of the flow response after the HV pulse application. The delay $\Delta t$ refers to the period of time between the HV pulse and the end of the camera acquisition gate 
(last possible time recorded by the camera). The burst cycle is repeated every $500 \mathrm{~ms}$ (i.e. at $2 \mathrm{~Hz}$ ). The $2 \mathrm{~Hz}$ burst frequency was primarily chosen due to the framerate limitation of the CCD camera used, to ensure a proper camera response. We must underline that, for the gas flow range investigated in this work, even a $100 \mathrm{~ms}$ time delay between two HV pulses is still long enough to avoid the accumulation of two perturbations in the same schlieren images (i.e. there is no interference between the last and the first pulse of two successive burst. Thus, series of schlieren images made at $2 \mathrm{~Hz}$ were used to verify the reproducibility of the phenomenon visualized. For each condition, a series of 20 schlieren images was taken. All the schlieren images shown in the present paper are not accumulated and one image is chosen over the series of 20 schlieren images.

For plasma plume imaging, a PI-MAX $4^{\circledR}$ intensified CCD (ICCD) camera was used, trigged by the DG 535. The gate delay and integration time were controlled by Lightfield software. $A$ Nikon objective was mounted on the ICCD camera (AF MICRO NIKKOR 60mm/1:2.8D) allowing, in our conditions, a resolution of $0.032 \mathrm{~mm} / \mathrm{pxl}$ with an error of $2 \%$. For plasma visualization with the ICCD camera, gates of 500 ns duration were used, covering the temporal region between $0 \mathrm{~ns}$ and $500 \mathrm{~ns}$ in Figure 2(b.). The ICCD gate was delayed in order to integrate over a given HV pulse in the series of HV pulses (first, second or any others). All waveforms are recorded with a LECROY WaveRunner 64Xi-A (600MHz 10Gs/s). The ICCD camera for plasma plume visualization and the CCD camera for schlieren visualization did not run simultaneously, to avoid excessive air perturbation when schlieren images were recorded. We must also underline that in this case, even if air movement is visible in the background of the schlieren images, the argon flow is not disturbed by the fan of the ICCD camera. As for schlieren images, series of plasma plumes with 20 images were acquired for each condition. Except for Figure 3(c.), all the plasma plumes shown in the present paper result from the numerical accumulation of 20 plasma plume images. For each image (CCD and ICCD), the contrast is maximized using the whole range of the image intensity value. For ICCD images, with the colormap used, the brighter and darker regions appear in yellow and black, respectively.

\section{Results and discussion}

\subsection{Single HV shot and double HV shots operation}

Figure 3(a.) shows schlieren images of the argon flow expansion downward the capillary outlet, between 800 and $400 \mathrm{sccm}$, without plasma activation. The transition point from laminar to turbulent regime (referred as "turbulent transition point" in the following) is measured on the images, at the end of the laminar region of the flow. The turbulent transition point distance is defined considering the origin at the capillary outlet (visible in black on the top of each image). The distance of the turbulent transition point is shown in Figure $\mathbf{3}(\mathbf{b}$.) (black squares) for each flow rate, resulting from the average of measurements on 20 different images. At $800 \mathrm{sccm}$, the turbulent transition point is located at $23.7 \pm 1.6 \mathrm{~mm}$. When the flow rate decreases, from 800 to $600 \mathrm{sccm}$, the turbulent transition point is gradually shifted downwards (to $28.9 \pm 1.7 \mathrm{~mm}$ at $700 \mathrm{sccm}$ and $38.0 \pm 2.6 \mathrm{~mm}$ at $600 \mathrm{sccm}$ ). It corresponds to 
the appearance of the established turbulence regime. At $500 \mathrm{sccm}$, the turbulent transition point is strongly shifted to $67.0 \pm 5.7 \mathrm{~mm}$. The flow is in a transitional regime and the fluctuation of the position of the turbulent transition point is more severe. It should be pointed out that the error of the measurement is almost negligible (about $200 \mu \mathrm{m}$ ) compared to the fluctuation of the position of the turbulent transition point. At $400 \mathrm{sccm}$, the flow is in a laminar regime in the full visualization area (length of $91.7 \mathrm{~mm}$ ). Figure $\mathbf{3}(\mathbf{c}$.) shows the emission of the plasma plumes in the same flow rate range, for single HV shot operation. It is worth mentioning that, in this flow rate range, the plasma plume expansion (its maximum average length is plotted in red circles in Figure $\mathbf{3}(\mathbf{b}$.$) ) is not limited by the position of the$ turbulent transition point, and benefits from the whole laminar region of the argon flow (except at $800 \mathrm{sccm}$, compare red circles and black squares in Figure 3(b.)). Hence, it results in stable plasma plumes, as shown in Figure 3(d.), for each flow rate, with the numerical accumulation of 20 plasma plumes, each obtained in single HV shot operation. Comparing Figure 3(c.) and Figure $\mathbf{3}(\mathbf{d}$.), it should be noted that the averaging process hides the filamentary aspect of the plumes. This is due to the change of the position of the filaments between each HV shot. This pronounced filamentary aspect is characteristic of plumes of argon plasma jet [20], compared to those obtained with helium, which are rather diffuse and homogeneous [17].

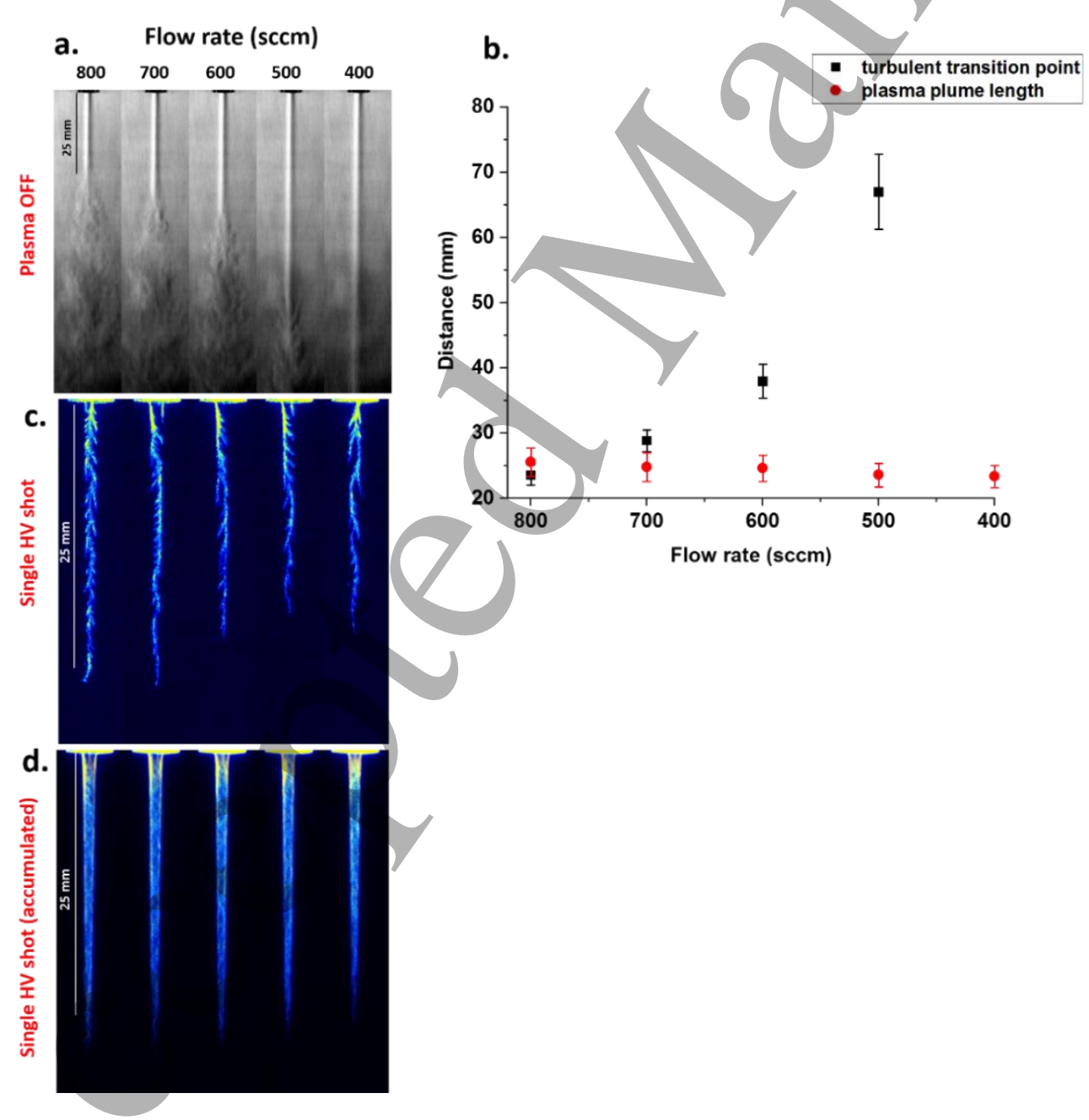

Figure 3 : Schlieren images of the argon flowing downward the capillary outlet in the range 800-400 sccm, without plasma activation (a.). Evolution of the position of the turbulent transition point and the length of the plasma 
plume downstream of the capillary outlet, with respect to the flow rate (b.). Plasma plumes emission in single HV shot operation in the same flow rate range, without (c.) and with (d.) numerical accumulation (series of 20 images per flow rate condition). The amplitude of the applied voltage is $6 \mathrm{kV}$.

Figure 4(a.) shows time-resolved schlieren images of the dynamics of the argon flow expansion, after the application of a single HV pulse (single HV shot operation). The delay $\Delta t$ refers to the period of time between the HV pulse and the end of the camera acquisition gate, as described in section 2. At $\Delta \mathrm{t}=0.85 \mathrm{~ms}$, the argon flow is similar to the plasma OFF case depicted in Figure 3(a.). At $\Delta t=1.70 \mathrm{~ms}$, the flow structure visible on the image appears essentially not impacted, except for a slight deformation with a small gas expansion, which is barely visible on the left side of the flow laminar region (indicated by an arrow at $12.3 \mathrm{~mm}$ ). At $\Delta \mathrm{t}=2.90 \mathrm{~ms}$, a ripple is visible inside the laminar region (also indicated by an arrow). Upstream, argon spreads from the laminar region (referred as "flow breakdown" in the following). The flow breakdown is fully developed at $3.50 \mathrm{~ms}$, and argon flow spreads while it is entrained downwards at 4.50 and $6.50 \mathrm{~ms}$.

\section{a. \\ Single HV shot}

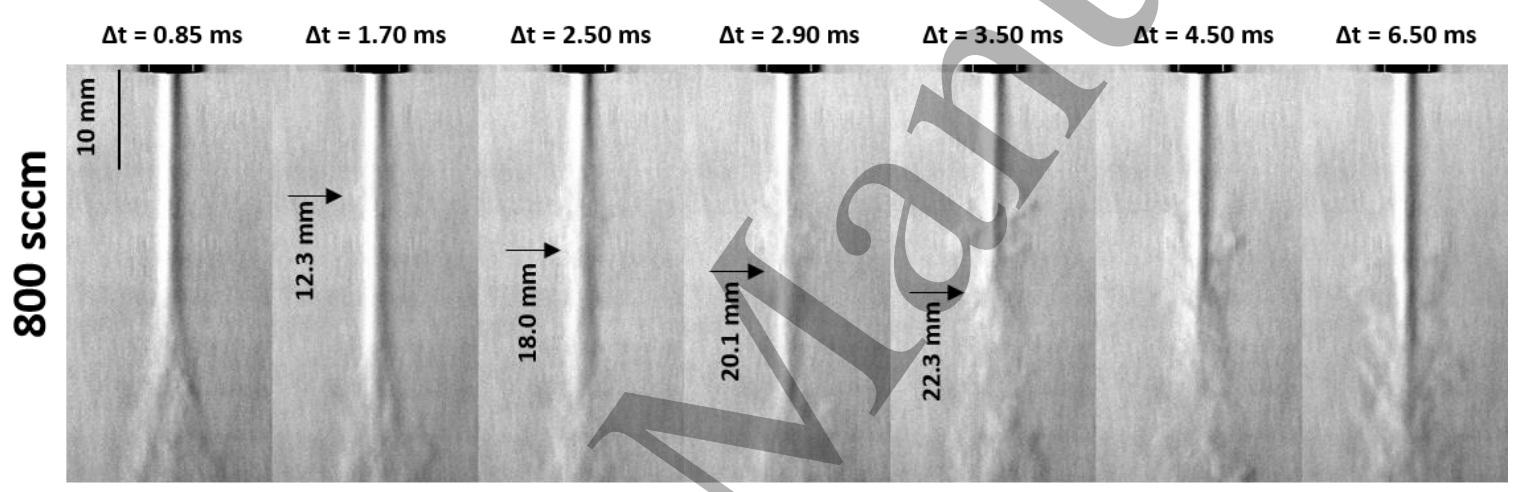

Double HV shots

b. $\Delta t=0.85 \mathrm{~ms} \Delta \mathrm{t}=1.70 \mathrm{~ms} \Delta \mathrm{t}=2.50 \mathrm{~ms} \Delta \mathrm{t}=2.90 \mathrm{~ms} \Delta \mathrm{t}=3.50 \mathrm{~ms} \Delta \mathrm{t}=4.50 \mathrm{~ms} \Delta \mathrm{t}=6.50 \mathrm{~ms}$

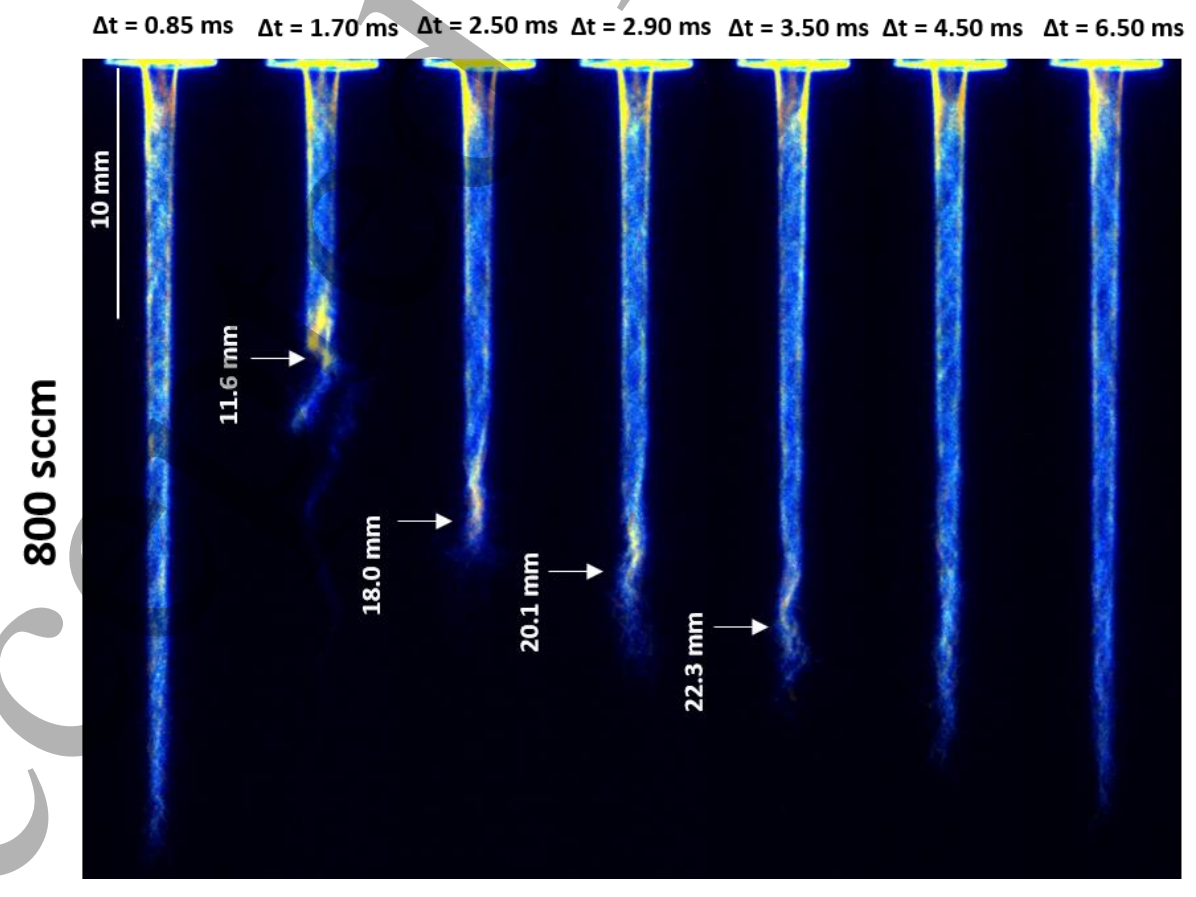


Figure 4 : Schlieren images of the argon flow dynamics at $800 \mathrm{sccm}$, after the application of one HV pulse (a.). The delay $\Delta t$ refers to the time interval between the HV pulse and the end of the camera acquisition gate. Plasma plumes emission in double HV shots operation at $800 \mathrm{sccm}$ (b.). The plasma emission is integrated over the second HV pulse (500 ns), and the delay $\Delta t$ between the two HV pulses is equal to that used for schlieren image acquisition. The plasma plumes result of the numerical accumulation of 20 images for each delay. The amplitude of the applied voltage is $6 \mathrm{kV}$.

Figure $\mathbf{4}$ (b.) shows the plasma plumes emission in double HV shots operation. As the drift velocity of the flow perturbation is extremely slow compared to the IW propagation velocity ( $\sim 10 \mathrm{~m} / \mathrm{s}$ vs $10^{5} \mathrm{~m} / \mathrm{s}$, respectively), the flow perturbation generated after the application of the second HV pulse has no time to drift while the IW propagates. Then, the IW of the second $\mathrm{HV}$ pulse can be used as a probe to highlight the flow structure resulting from the application of the first HV pulse. The ICCD camera integrates plasma emission over the second HV pulse and the delay $\Delta t$ between the first and the second HV pulses corresponds exactly to the value of $\Delta t$ given in Figure 4(a.). As for Figure 3(d.), the plasma plumes are numerically accumulated over $20 \mathrm{HV}$ pulses. Interestingly, the plasma plume is already severely affected at $\Delta \mathrm{t}=1.70 \mathrm{~ms}$, when compared to the plume at $\Delta t=0.85 \mathrm{~ms}$. This results in a limited plasma plume length, with a twisted plasma expansion at the tip (corresponding to the beginning of the flow breakdown observed on the schlieren image for the same delay). The plasma is also characterized by a brighter emission at the tip. Afterwards, the length of the plasma plume gradually increases over time, and the full length is recovered around $4.50 \mathrm{~ms}$. This observation is of importance because it shows that the flow structure is already affected by the ignition of a single discharge event. Considering that the perturbation is generated at the inner electrode tip inside the capillary when the discharge is ignited, the drift velocity is 12.7 $\mathrm{m} / \mathrm{s}$ (i.e. $21.6 \mathrm{~mm}$ traveled in $1.70 \mathrm{~ms}$, taking the position of the plasma plume tip just before the twisted region). This value is within $10 \%$ of the value of the mean flow velocity, estimated to be $11.8 \mathrm{~m} / \mathrm{s}$, given that $800 \mathrm{sccm}$ of argon flow through a $1.2 \mathrm{~mm}$ inner diameter hollowed needle. Hence, the flow perturbation is likely to be generated at the inner electrode and then entrained by the flow. The time to recover the full length of the plasma plume is consequently explained by the time required to evacuate the perturbation. Furthermore, it should be noted that the plasma plume is longer at $0.85 \mathrm{~ms}$ in Figure $\mathbf{4 ( b . )}(30.5 \pm 1.4 \mathrm{~mm}$ ) than in $\mathbf{3}$ (d.) for 800 $\mathrm{sccm}(25.6 \pm 2.2 \mathrm{~mm} \mathrm{~mm})$ or at $6.50 \mathrm{~ms}$ in Figure 4 (b.) $(27.3 \pm 1.8 \mathrm{~mm})$. At the first HV shot, the instantaneous voltage corresponding to the IW ignition at the inner electrode (not shown) is $5.4 \mathrm{kV}$, which is close to the maximum amplitude of the $\mathrm{HV}$ pulse $(6 \mathrm{kV})$ according to its time evolution (see Figure 2(b.)). With a $0.85 \mathrm{~ms}$ delay between the two HV shots, the second IW is ignited at $3.7 \mathrm{kV}$ (32\% smaller than for the first IW), still in the rising part of the HV pulse. This could explain why the plasma plume at $\Delta \mathrm{t}=0.85 \mathrm{~ms}$ is significantly longer (+19\%) at double shots than at single shot operation, because, as the second IW initiates earlier, it has consequently more time to propagate, due to the HV pulse temporal evolution. With a mean flow velocity of $11.8 \mathrm{~m} / \mathrm{s}$, the perturbation generated at the first IW ignition should be located close to the capillary outlet at $\Delta \mathrm{t}=0.85 \mathrm{~ms}$ (the distance between the hollowed needle and the capillary outlet is $10 \mathrm{~mm}$, as shown in Figure $\mathbf{1}$ (a.)). At $\Delta t=6.50 \mathrm{~ms}$, the perturbation has been fully evacuated and the plasma plume retrieves a length comparable to that of the first shot in Figure 3(d.). As a matter of fact, on the timescale of the IW propagation, the flow can be considered as static, and, thus, the flow perturbation generated after the first HV pulse application is static while the second IW propagates. When the second IW is launched at 
$\Delta \mathrm{t}=6.50 \mathrm{~ms}$, it propagates in a non-perturbated flow as the perturbation had been evacuated and, thus, its time and voltage of ignition is similar to those of an IW at single shot operation or of the first IW at double shots operation. However, when the second IW is launched and the perturbation is still located inside the capillary or outside it but in the close vicinity of its outlet (at $\Delta \mathrm{t}=0.85 \mathrm{~ms}$, for instance), its time and voltage ignition are clearly lower. This difference on the ignition time at the second HV pulse could hardly be explained by the remaining electron density after the first IW propagation, due to the timescale involved (850 $\mu \mathrm{s})$. Indeed, for an argon streamer in argon, Hübner et al. [73] have measured that the electron density falls down to $1 \times 10^{12} \mathrm{~cm}^{-3}$ after $1.1 \mu \mathrm{s}$, which is almost three orders of magnitude shorter. Moreover, the charge deposition on the capillary wall, after only one IW generation, is expected in the order of only few $\mathrm{nC}[74]-[78]$.

Table 1 resumes the position of the brighter regions of the plasma plume tips, associated to the flow perturbation, with respect to the delay. The corresponding perturbation drift velocities are also indicated. At $1.70 \mathrm{~ms}$, the velocity is estimated taking into account the distance between the tip of the hollowed needle (HV electrode, where the discharge is ignited) and the position indicated on the plasma plume region in Figure 4(b.). From 2.50 to $3.50 \mathrm{~ms}$, the velocities are estimated between two successive positions indicated on the plasma plume images of Figure $\mathbf{4}(\mathbf{b}$.$) .$

\begin{tabular}{|c|c|c|}
\hline$\Delta \mathrm{t}(\mathrm{ms})$ & $\begin{array}{c}\text { Bright plasma plume tip } \\
\text { position }(\mathbf{m m})\end{array}$ & $\begin{array}{c}\text { Flow perturbation drift } \\
\text { velocity }(\mathbf{m} / \mathbf{s})\end{array}$ \\
\hline 1.70 & 11.6 & 12.7 \\
\hline 2.50 & 18.0 & 8.0 \\
\hline 2.90 & 20.1 & 5.3 \\
\hline 3.50 & 22.3 & 3.7 \\
\hline
\end{tabular}

Table 1 : Flow pertubation positions and drift velocities estimated from the positions of the brighter regions of the plasma plume tips indicated in Figure 4 (b.) for different delays $\Delta t$.

The evolution of the length of the plasma plume directly indicates the flow velocity profile downstream of the capillary outlet. The argon flow velocity is close to the mean flow velocity through the hollowed needle (inner diameter) on the first $12 \mathrm{~mm}$. It may correspond to the region of the potential core, which is rigorously defined by a homogenous concentration of the fluid jet and a uniform velocity profile along the jet radius. Then, as the flow expands downstream, it is slowed down while mixing with ambient air. Also, at 4.50 and $6.50 \mathrm{~ms}$, the IW is beyond the turbulent transition point.

Similarly to Figure 4(b.), Figure 5 shows plasma plumes in double HV shots operation, but before the delay $\Delta t=1.70 \mathrm{~ms}$ and the associated brutal reduction of the plasma plume length. At $1.00 \mathrm{~ms}$, a ripple is clearly visible in the plasma plume. The amplitude of the ripple increases while it moves downwards, but the length of the plasma plume is not affected. The rippled regions are also characterized by a brighter plasma emission. Note that the bright regions are present at each HV pulse, and are not the result of the accumulation over $20 \mathrm{HV}$ pulses. From $1.15 \mathrm{~ms}$, it can be observed that the diameter of the plasma plume starts to shrink in the rippled region. Finally, at $1.45 \mathrm{~ms}$, the deformation constrains the plasma plume expansion, and its length is reduced. At this delay, among the plasma plumes acquired over the series of 
$20 \mathrm{HV}$ pulses, only few plasma plumes were brutally stopped. It consequently corresponds to a delay close to the very beginning of the flow breakdown (precisely measured to start at 1.40 $\mathrm{ms}$, when only one plasma plume was stopped over the series of HV pulses).

Figure 6(a.) shows the emission of the plasma plumes during the rippled phase (before the flow breakdown) for flow rates between 800 and $400 \mathrm{sccm}$ (step of $100 \mathrm{sccm}$ ). The plasma plumes are imaged with the appropriate delay $\Delta t$, in order to visualize the ripple few hundreds of $\mu$ s after its appearance (the delays are resumed in Table 2). From 800 to $500 \mathrm{sccm}$, the ripple is well visible. The delay of appearance and the position of the ripple are sensitive to the flow rate. When the flow rate decreases, the delay of appearance increases, and the position (pointed by white arrows Figure 6(a.)) is shifted downwards (resumed in Table 2). It should also be noted that the amplitude of the deformation is also sensitive to the flow rate. When the flow rate decreases, the amplitude of the deformation is also reduced. At $400 \mathrm{sccm}$, the ripple is still present, but barely visible. Moreover, the intensity of the plasma emission in the rippled region is also affected by the flow rate. While the plasma emission is more intense in the rippled region from 800 to $500 \mathrm{sccm}$ (even if the emission intensity decreases with decreasing flow rate), this is not the case for $400 \mathrm{sccm}$. These differences on the ripple characteristics have also a consequence on the onset of the flow breakdown, as shown in Figure $\mathbf{6}(\mathbf{b}$.). The delays were chosen in order to visualize the very beginning of the flow breakdown, as described when discussing the latest delay of Figure 5. From 800 to $600 \mathrm{sccm}$, the ripple still induces a flow breakdown with a narrowed plasma plume. But, from $500 \mathrm{sccm}$ down, the deformation is not strong enough to trigger the flow breakdown, even if it is still visible (barely at $400 \mathrm{sccm}$ ). It consequently appears that for exactly the same perturbation event (the discharge ignition), the flow structure is differently affected, depending on the flow rate.

Double HV shots

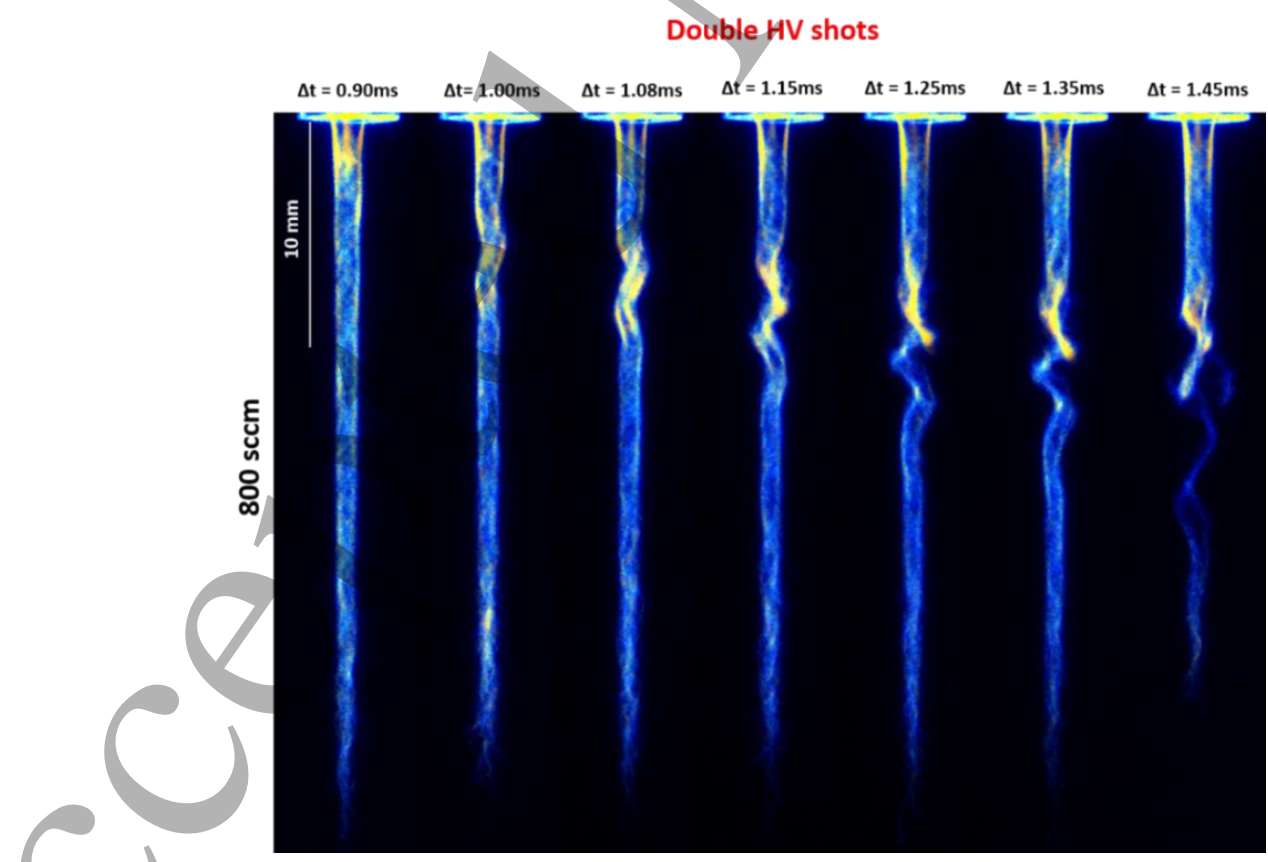

Figure 5 : Plasma plumes emission in double HV shots operation at $800 \mathrm{sccm}$. The plasma emission is integrated over the second HV pulse (500 ns), and $\Delta t$ corresponds to the delay between the two HV pulses. The plasma plumes result from the numerical accumulation of 20 images, for each delay. The amplitude of the applied voltage is $6 \mathrm{kV}$. 
a.

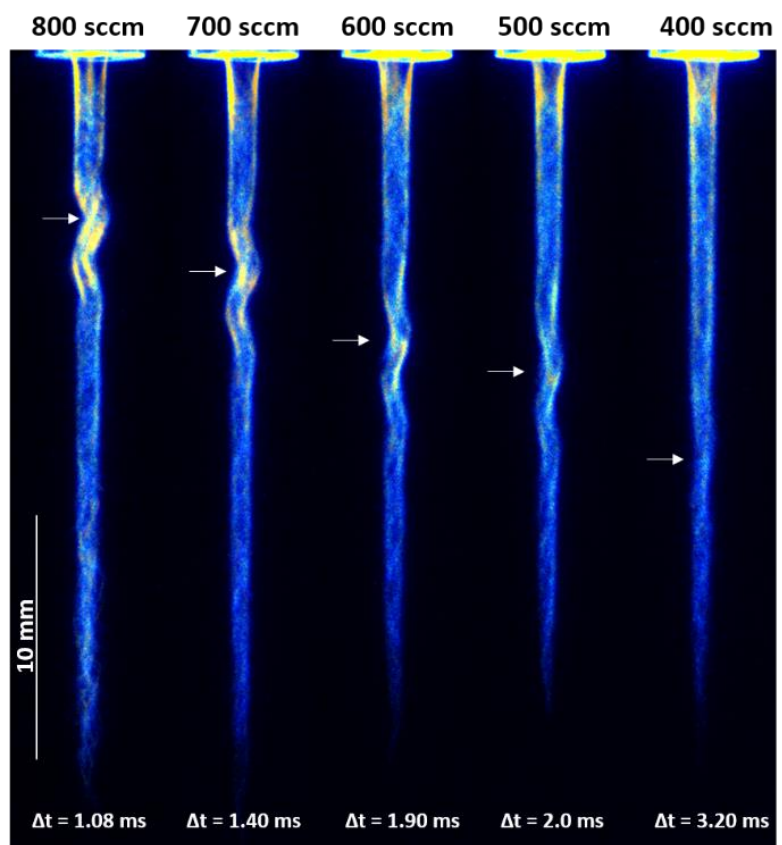

b.

Double HV shots

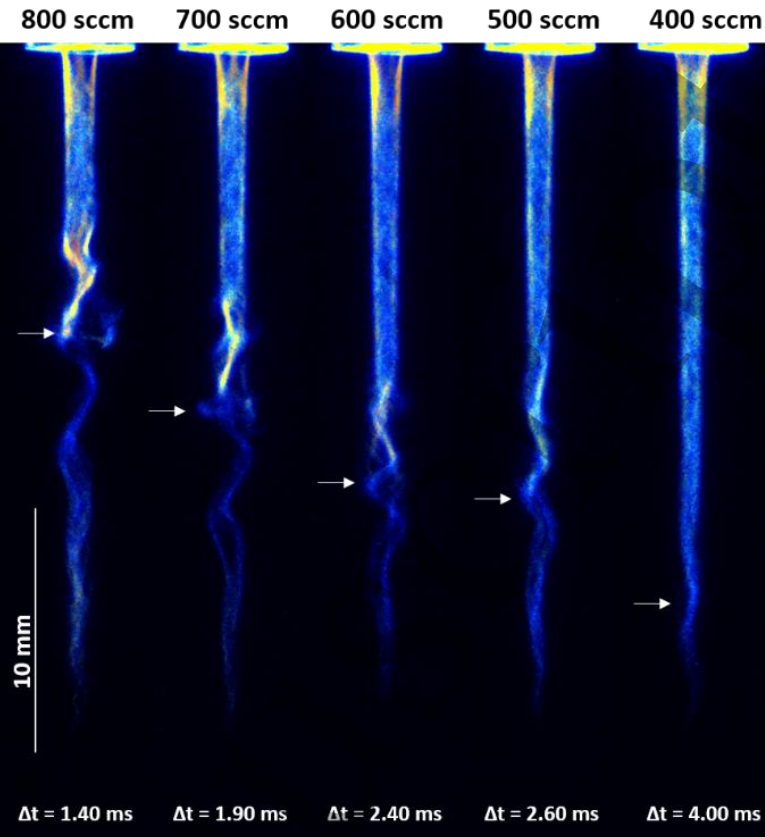

Figure 6 : Plasma plumes emission in double HV shots operation, during the rippled phase (a.) and the flow breakdown phase (b.), from 800 to $400 \mathrm{sccm}$ (step of $100 \mathrm{sccm}$ ). The plasma emission is integrated over the second HV pulse (500 ns), and $\Delta$ t corresponds to the delay between the two HV pulses. The plasma plumes result from the numerical accumulation of 20 images, for each delay. The amplitude of the applied voltage is $6 \mathrm{kV}$.

Table 2 resumes, for each flow rate, the delay of appearance of the ripple and its position, the corresponding propagation velocity and the relative difference to the mean flow velocity.

\begin{tabular}{|c|c|c|c|c|c|c|}
\hline $\begin{array}{c}\text { Flow } \\
\text { rate } \\
(\mathbf{s c c m})\end{array}$ & $\begin{array}{c}\text { Delay of } \\
\text { appearance } \\
\text { of the ripple } \\
\text { after the HV } \\
\text { pulse } \\
\text { application } \\
\text { (ms) }\end{array}$ & $\begin{array}{c}\text { Distance } \\
\text { from the } \\
\text { hollowed } \\
\text { needle tip } \\
(\mathbf{m m})\end{array}$ & $\begin{array}{c}\text { Propagation } \\
\text { velocity of } \\
\text { the } \\
\text { perturbation } \\
(\mathbf{m} / \mathbf{s})\end{array}$ & $\begin{array}{c}\text { Mean flow } \\
\text { velocity } \\
\text { (hollowed } \\
\text { needle } \\
\text { inner } \\
\text { diameter) } \\
\text { (m/s) }\end{array}$ & $\begin{array}{c}\text { Velocities } \\
\text { relative } \\
\text { difference }\end{array}$ & $\begin{array}{c}\text { Re } \\
\text { number } \\
\text { (hollowed } \\
\text { needle } \\
\text { inner } \\
\text { diameter) }\end{array}$ \\
\hline $\mathbf{8 0 0}$ & 0.93 & $\mathbf{1 3 . 9}$ & 14.9 & 11.8 & $21 \%$ & 1241 \\
\hline $\mathbf{7 0 0}$ & 1.10 & $\mathbf{1 4 . 9}$ & 13.5 & 10.3 & $24 \%$ & 1086 \\
\hline $\mathbf{6 0 0}$ & 1.30 & $\mathbf{1 5 . 8}$ & 12.2 & 8.8 & $28 \%$ & 930 \\
\hline $\mathbf{5 0 0}$ & 1.90 & $\mathbf{1 9 . 6}$ & 10.3 & 7.4 & $28 \%$ & 775 \\
\hline $\mathbf{4 0 0}$ & 3.0 & $\mathbf{2 6 . 4}$ & 8.8 & 5.9 & $33 \%$ & 620 \\
\hline
\end{tabular}

Table 2 : Delay of appearance and position of the ripple after the HV pulse application, measured on plasma plumes images (not shown) numerically accumulated over $20 \mathrm{HV}$ pulses. The corresponding perturbation propagation velocities are compared to the mean flow velocities. The corresponding Reynolds number (Re) are also given, with the argon density at atmospheric pressure and ambient temperature.

For all flow rates, it appears that the propagation velocity of the perturbation generated when the HV pulse is applied is higher than the mean flow velocity (between $21 \%$ and $33 \%$ ). It should be also pointed out that these mean flow velocities represent high values (inner diameter of the hollowed needle considered), which are likely overestimated in the concerned 
regions for 500 and $400 \mathrm{sccm}(9.6 \mathrm{~mm}$ and $16.4 \mathrm{~mm}$ downstream of the capillary outlet, respectively).

Figure 7 shows schlieren images of the argon flow from 800 to $400 \mathrm{sccm}$, but for later delays after the HV pulse application. These images show that, for all the flow rates, the ripple continues to propagate in the laminar flow structure (pointed by black arrows), once it has passed the flow breakdown point. For the higher flow rates ( 800 and $700 \mathrm{sccm}$ ), the distance between the point of flow breakdown and the turbulence transition point is small, which reduces the time interval to observe the ripple propagation after the flow breakdown point. Working with moderate flow rates makes the observation easier, because the laminar region is much longer. It appears clearly that the lower the flow rate, the farther the time interval of appearance of the ripple and, consequently, the longer the distance travelled by these flow deformations. As the ripples propagate, their amplitude increases. This increase in amplitude is especially visible at low flow rates, as the ripples are present over a longer period of time. The very moderate impact on the plasma plumes at $400 \mathrm{sccm}$ seen in Figure 6 is explained by the fact the necessary amplitude to significantly disturb the plasma plume occurs far downstream from the plasma plume region. Consequently, the flow modification mechanism cannot be explained by ionic wind action or direct heating in the plasma plume region. The modification operates through a perturbation, which is likely generated in the early phase of the discharge development inside the capillary, when the IW is ignited at the inner electrode. The origin of this perturbation has been described by Lietz et al. [44], for helium CAPPJ. The authors reported that one IW ignition results in a fast temperature increase in the HV electrode region, which results in doubling the pressure in this region. The authors have also shown that the resulting acoustic wave propagates and disturbs the unstable helium shear layer, downstream of the capillary outlet. It is likely that, in our experimental conditions, with a pulsed argon atmospheric pressure plasma jet, the origin of the observed perturbation also lies in a pressure increase close to the tip of the hollowed needle due to a local fast heating resulting from the IW ignition. However, in our case with an argon plasma jet, the flow is significantly perturbated despite of the fundamentally less unstable features of the argon shear layer [54]. According to Table 2, the pressure wave propagates at a group velocity, which is significantly higher than the mean flow velocity. More details on the perturbation mechanism, with consideration of the gas injection geometry, are given in the section 3.3. The flow modification appears, then, as a direct consequence of the relaxation of this perturbation. For a single IW event, the relaxation time and the observed flow deformation directly depend on the flow rate value. To the best of our knowledge, it is the first time that such phenomenon with a single HV shot approach is experimentally evidenced for an atmospheric pressure plasmajet. Also, to the extent that the IW propagation itself does not affect the flow structure (only its ignition does), the double HV shots technique can be directly used to probe the flow structure, by varying the time delay between the two HV pulses and surveying the light emission of the second IW. This allows to instantaneously image the argon flow in a non-intrusive way, tracking very precisely the dynamic of the flow perturbation generated after the first IW ignition. 


\section{Single HV shot}

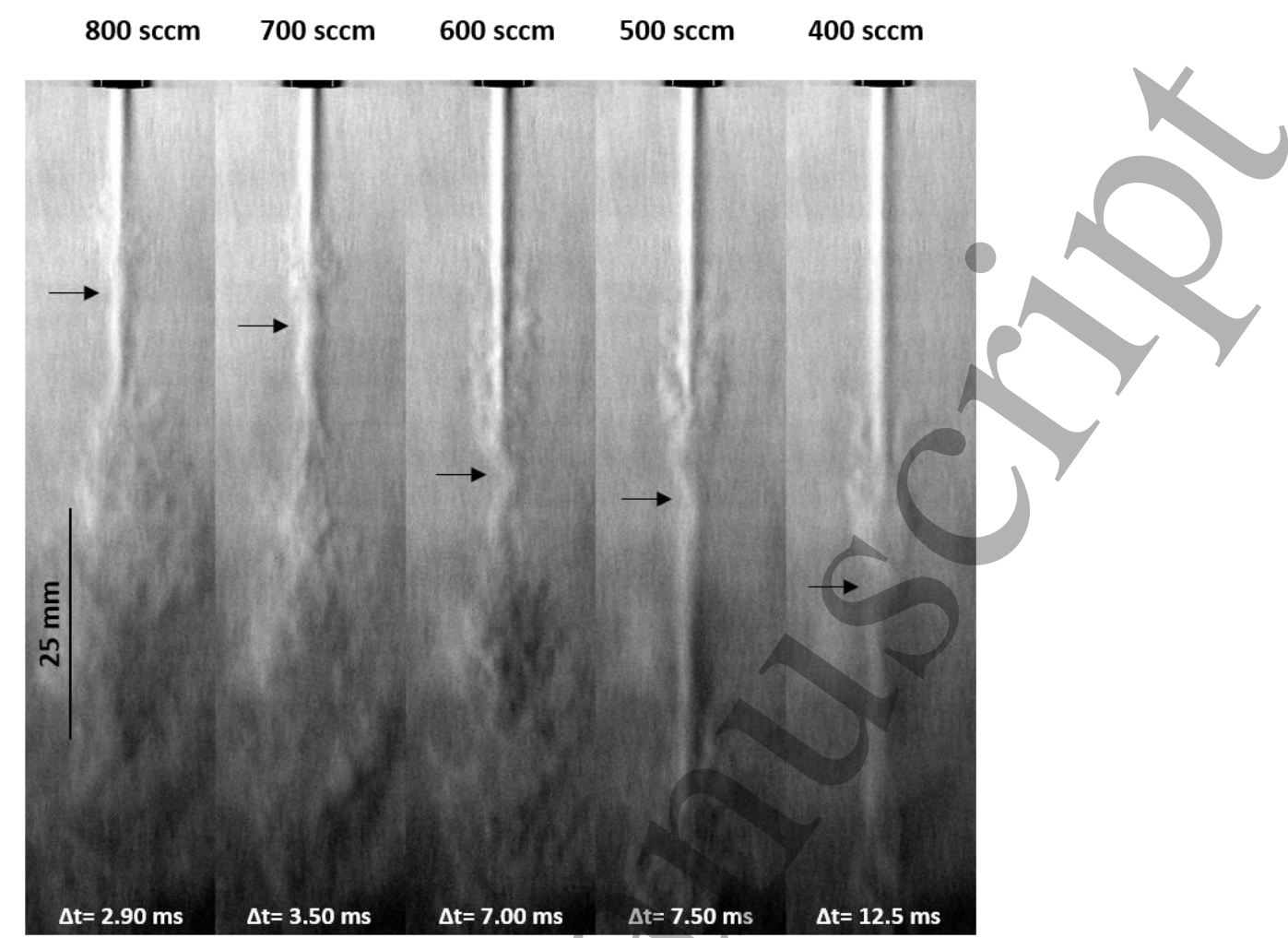

Figure 7 : Schlieren images of the argon flow after the application of one HV pulse for flow rates between 800 and $400 \mathrm{sccm}$. The delays $\Delta t$ refer to the time interval between the HV pulse application and the end of the camera acquisition gate. The amplitude of the applied voltage is $6 \mathrm{kV}$.

\subsection{Multiple HV shots operation}

Due to the timescale of the complete relaxation of the flow after the application of one HV pulse, memory effects are strongly expected when the plasma jet is used in pulsed repetition mode. Figure 8 shows schlieren images of the argon jet when a series of HV pulses are applied at PRF between 1.5 and $10 \mathrm{kHz}$. For each flow rate, the delay $\Delta \mathrm{t}$ always refers to the time interval between the application of the first HV pulse of the series and the end of the camera acquisition gate. For a given PRF, the number of HV pulses applied $n$ is given by $n=$ $\Delta t \times P R F+1$. Consequently, the higher the PRF, the higher the number of HV pulses applied before the end of the camera acquisition gate. For each flow rate, the delay $\Delta t$ is chosen in order to visualize the flow after the flow breakdown ignition in single HV shot operation. 


\section{Multiple HV shots}

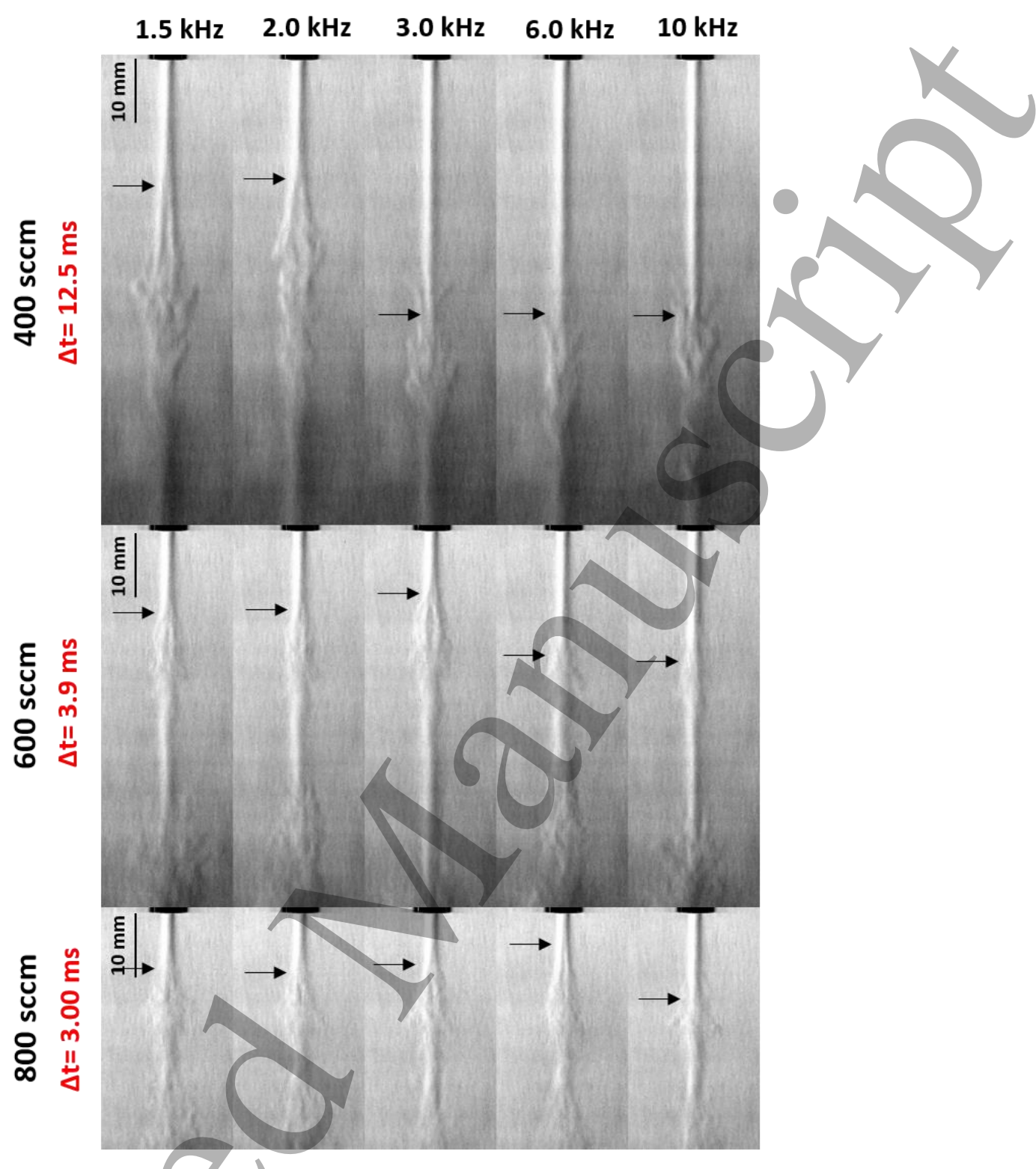

Figure 8 : Schlieren images of the argon flow between 800 and $400 \mathrm{sccm}$ (step of $200 \mathrm{sccm}$ ), with HV pulses repetitively applied at PRF between 1.5 and $10 \mathrm{kHz}$. The delay $\Delta t$ refer to the time interval between the application of the first HV pulse and the end of the camera acquisition gate. The amplitude of the applied voltage is $6 \mathrm{kV}$.

At $400 \mathrm{sccm}$ and $1.5 \mathrm{kHz}$, the flow exhibits a radial splitting, with a net separation between two jets in the laminar region. The flow perturbation point, corresponding to the beginning of the radial splitting is marked by a black arrow. It highly contrasts with the flow structure in single HV shot operation at the same delay, shown in Figure 7. At $2.0 \mathrm{kHz}$, the radial splitting slightly moves upwards, but it is still well visible. Surprisingly, at $3.0 \mathrm{kHz}$, the radial splitting disappears, and the flow retrieves a long straight expansion. Then, the flow structure remains globally the same at 6.0 and $10 \mathrm{kHz}$, without radial splitting. At $600 \mathrm{sccm}$, the radial splitting is visible from 1.5 to $3.0 \mathrm{kHz}$. The flow perturbation point gradually moves upwards, and the radial expansion increases. For higher $\mathrm{PRF}$, here shown at 6.0 and $10 \mathrm{kHz}$, the radial splitting disappears, and the flow perturbation point moves downwards. Finally, at $800 \mathrm{sccm}$, the radial splitting still occurs at $6.0 \mathrm{kHz}$ and is not visible at $10 \mathrm{kHz}$. It can also be observed that, from 
1.5 to $6.0 \mathrm{kHz}$, the flow breakdown point gradually moves upwards, and then downwards at $10 \mathrm{kHz}$.

Figure 9 shows the detailed evolution of the mean flow perturbation point, measured as the distance to the capillary outlet, with respect to the HV PRF, for flow rates between 400 and $800 \mathrm{sccm}$. The position is manually measured on each schlieren image, and it corresponds to the beginning of the change of the diameter of the straight flow region. Each point results from the average of measurements on 20 different schlieren images.
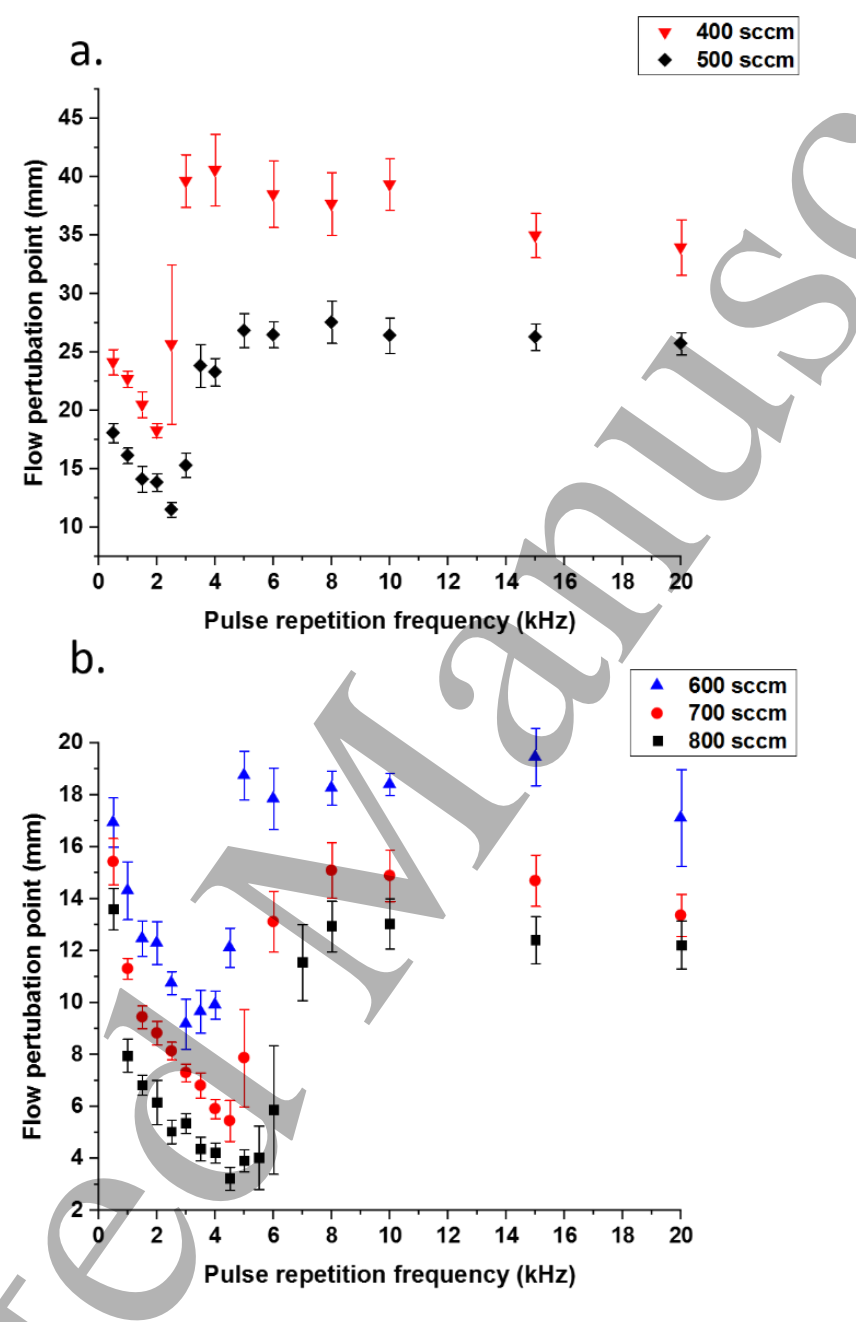

Figure 9 : Evolution of the flow perturbation point with the HV PRF for flow rates between 400 and $800 \mathrm{sccm}$ (step of $100 \mathrm{sccm}$ ). Each point results from the average of measurements on 20 different schlieren images.

At $400 \mathrm{sccm}$, the mean flow perturbation point progressively moves upwards from 0.5 to 2 $\mathrm{kHz}$ and is well localized $(24.1 \pm 1.1 \mathrm{~mm}$ and $18.3 \pm 0.6 \mathrm{~mm}$, respectively). It corresponds to the development of the radial splitting depicted in Figure 8. At $2.5 \mathrm{kHz}$, the flow perturbation point starts to move downwards, and the radial splitting has almost vanished. The flow structure alternates from one image to another between straight expansion and a barely visible splitting, which increases the fluctuation of the flow perturbation point ( $25.6 \pm 6.8 \mathrm{~mm})$. At $3 \mathrm{kHz}$, the radial splitting has completely vanished (straight flow expansion in Figure 8), and the flow perturbation point is strongly shifted downwards $(39.6 \pm 2.3 \mathrm{~mm})$. Then, for higher PRF, a plateau is reached, and the flow perturbation point remains in the same position within 
the fluctuations. At $500 \mathrm{sccm}$, the maximal development of the radial splitting occurs at 2.5 $\mathrm{kHz}(11.5 \pm 0.6 \mathrm{~mm})$. The splitting vanishes around $4 \mathrm{kHz}$, and the plateau with a straight flow expansion is reached around $5 \mathrm{kHz}(26.8 \pm 1.5 \mathrm{~mm})$. For the other higher flow rates, a similar behavior is observed with the increasing of the PRF. The radial splitting develops at low PRF, and the flow perturbation point moves upwards quasi-linearly with the PRF. With increasing PRF, the radial splitting gradually disappears, and the flow perturbation point moves downwards, before finally vanishing, when the straight flow expansion is reached. It appears clearly that when the flow rate increases, the PRF of transition and the PRF range of transition between the two phases (radial splitting and straight flow) also increase.

Table 3 resumes, for all the flow rates, the PRF associated with the maximum development of the radial splitting and with the appearance of the straight flow expansion, once the splitting has vanished. In the PRF range between those two PRF, the radial splitting gradually disappears.

\begin{tabular}{|c|c|c|}
\hline $\begin{array}{c}\text { Flow rate } \\
\text { (sccm) }\end{array}$ & $\begin{array}{c}\text { Radial splitting } \\
\text { maximum } \\
\text { development } \\
\text { (kHz) }\end{array}$ & $\begin{array}{c}\text { Appearance of the } \\
\text { straight flow } \\
\text { expansion } \\
\text { (kHz) }\end{array}$ \\
\hline $\mathbf{4 0 0}$ & 2.0 & 3.0 \\
\hline $\mathbf{5 0 0}$ & 2.5 & 4.0 \\
\hline $\mathbf{6 0 0}$ & 3.0 & 5.0 \\
\hline $\mathbf{7 0 0}$ & $4.0 / 4.5$ & 7.0 \\
\hline $\mathbf{8 0 0}$ & $4.5 / 5.0$ & 8.0 \\
\hline
\end{tabular}

Table 3 : PRF associated with the maximum development of the radial splitting and with the appearance of the straight flow expansion, for flow rates between 400 and $800 \mathrm{sccm}$.

These observations show that the effect of the PRF on the flow structure directly depends on the flow rate used. The PRF has also direct consequences on the plasma plumes shape, as it is illustrated in Figure 10, which shows plasma plume emissions at $600 \mathrm{sccm}$, for PRF between 0.5 and $20 \mathrm{kHz}$. For each PRF, the plasma emission is integrated over the HV pulse the closest to $\Delta t=3.9$ ms after the first HV pulse application, which corresponds to the $\Delta t$ of Figure 8 for $600 \mathrm{sccm}$. From 0.5 to $3.0 \mathrm{kHz}$, the plasma plume length decreases and the ripple alternation changes. The minimal length at $3.0 \mathrm{kHz}$ corresponds to the maximal development of the radial splitting depicted in Figure 8. With naked eyes, these plumes appear with stationary 3Dhelical-like structure. When the PRF increases, the progressive disappearance of the ripples corresponds to the gradual vanishing of the radial splitting, and the flow perturbation point moves downward. The PRF of the appearance of the straight plasma plume at $5 \mathrm{kHz}$ (see Figure 10) corresponds to the PRF of the appearance of the straight flow expansion in Table 3, also depicted in Figure 8. Once the flow exhibits a straight expansion, the flow perturbation (images at 6 and $10 \mathrm{kHz}$ in Figure 8) does not impact the plasma plume expansion (the reason behind it is discussed in the section 3.3). 


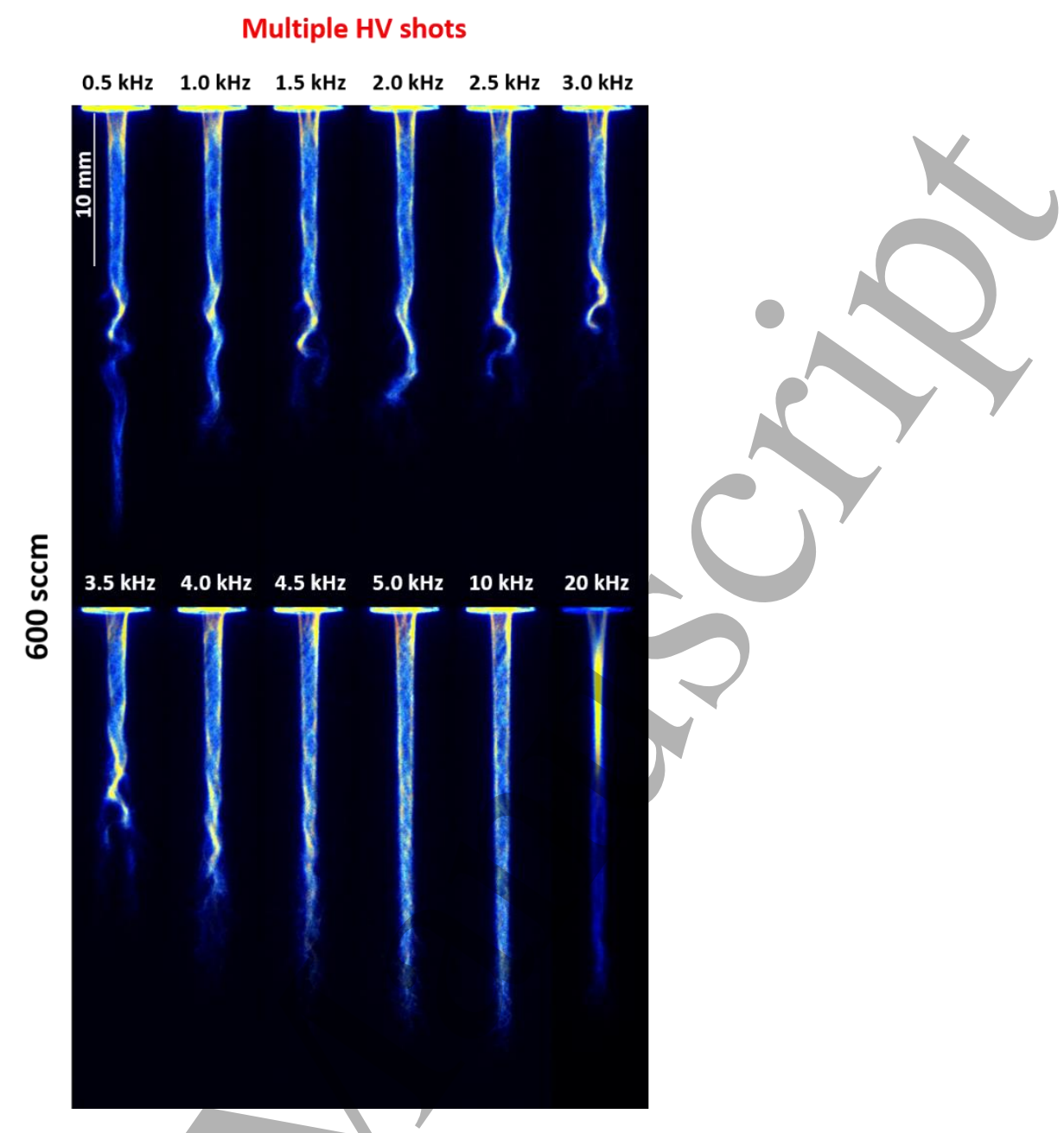

Figure 10 : Plasma plumes emission at 600 sccm for multiple HV shots operation, for PRF between 0.5 and $20 \mathrm{kHz}$. The plasma emission is integrated (500 ns) over the HV pulse the closest to $\Delta t=3.9 \mathrm{~ms}$ after the first HV pulse application. The plumes result from the numerical accumulation of 20 images. The amplitude of the applied voltage is $6 \mathrm{kV}$.

Table 4 resumes the PRF of the ripple disappearance in the plasma plumes for flow rates between 800 and $400 \mathrm{sccm}$.

\begin{tabular}{|c|c|}
\hline $\begin{array}{c}\text { Flow rate } \\
\text { (sscm) }\end{array}$ & $\begin{array}{c}\text { PRF of ripple disappearance } \\
\text { (straight plasma plume) } \\
\text { (kHz) }\end{array}$ \\
\hline 400 & 2.5 \\
\hline 500 & 3.5 \\
\hline 600 & 5.0 \\
\hline 700 & 6.5 \\
\hline 800 & 7.0 \\
\hline
\end{tabular}

Table 4 : PRF associated with the ripples' disaperance in the plasma plumes, for flow rates between 400 and 800 sccm.

The PRF of the ripples' disappearance can be found in the PRF range corresponding to the radial splitting gradual disappearance shown in Figure 9 . These phenomena cannot only be explained by the effect of the accumulation of multiple perturbations inside the gas flow. Otherwise, for a fixed delay $\Delta t$, the higher the number of HV pulses, the more affected the 
flow would be. Instead, beyond a precise transition PRF, depending on the flow rate, and corresponding to the PRF of the appearance of the straight flow expansion, the flow structure is globally reorganized and much less disturbed.

\subsection{Mechanism of flow modification}

The effect of the excitation PRF on the flow structure must be considered together with the specificity of the geometry of the gas injection used. Indeed, as shown in Figure 1, the hollowed needle's inner diameter and the capillary's inner diameter are of $1.2 \mathrm{~mm}$ and 1.7 $\mathrm{mm}$, respectively. Consequently, the argon flowing from the hollowed needle to the capillary undergoes a sudden expansion at their junction. Figure 11 sketches out the BFS flow field with the main regions delimited.

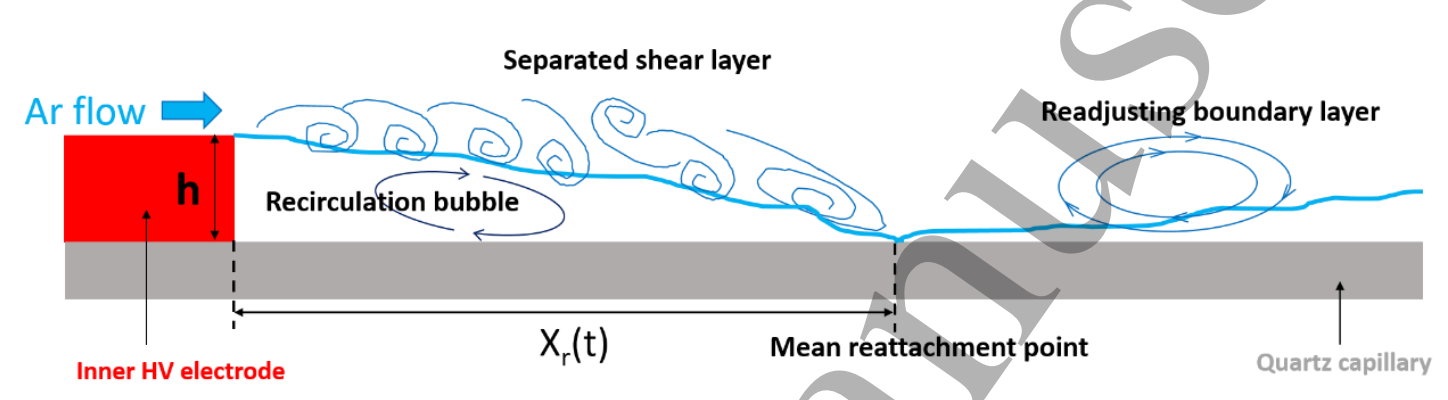

Figure 11 : Schematic 2D view of the backward-facing step flow field with the argon injection geometry of Figure 1(a.). These flow features do not depend on the nature of the gas used, for a same Re number. No HV pulse is applied on the inner HV electrode."

Under the effect of the adverse pressure gradient following the flow expansion, a recirculation bubble with a reversal flow is formed, right after the step. In this region, the maximum mean velocity of the reversal flow velocity could be about $20 \%$ of the free flow velocity [79]. Above the recirculation bubble, a separated shear layer is formed (mixing layer), with the roll-up of vortices by Kelvin-Helmholtz instability. Large coherent structures are formed and organized in a periodic manner [80]. The separated shear layer is curved and finally impacts the wall at the mean reattachment point. At this point, the flow structure is dominated by the large coherent structure and the position of the mean reattached point fluctuates in time due to the disparity of vortex size. Periodic impacts of the large scale structure on the wall is referred as the vortex shedding mode of the BFS flow [81]. Downstream of the reattachment point, the flow relaxes with the development of an internal boundary layer (called recovered or readjusting boundary layer). It has been observed that large scale coherent structures can survive in this region, far downstream from the reattachment point [80].

Several flow control strategies with different actuation techniques have been applied on BFS flow (see Table 4 in [62]), from external acoustic actuators to plasma actuators. By setting a surface DBD plasma actuator on the step corner in a BFS flow geometry, Benard et al. [82] have clearly shown that the mean reattachment point and flow instability modes can be changed with plasma periodic perturbations, according to plasma actuation parameters. The authors proceeded with a parametric study of the voltage amplitude, burst frequency and duty cycle. They have shown that the dynamic of the shear layer upstream and downstream 
of the mean reattachment point can be strongly influenced by the periodic perturbations imposed by the plasma actuator, when the excitation is tuned at the appropriate frequency compared to the flow instability mode. Under the plasma actuation, the authors have shown that the recirculation bubble and the mean reattachment point location can be minimized, the pressure fluctuation on the wall can be maximized and the vortex pairing can be enhanced downstream of the mean reattachment point. They have also shown that depending on the forcing mode, the pressure fluctuations on the wall and the mean reattachment point oscillate at the frequency imposed by the plasma actuator. Several other studies have also shown BFS flow control with plasma actuators [83]-[88].

What is quite remarkable for atmospheric pressure plasma jets with an inner hollowed HV electrode inserted inside a capillary is that the IW is naturally ignited at the triple point between the inner electrode, the rare gas and the capillary surface, which corresponds exactly to the recirculating bubble region of the BFS flow. Thus, it could be expected that the sudden pressure increase due to the IW ignition changes the force balance in the reattachment region of the BFS flow. Consequently, the periodic application of the HV pulses mechanically forces the flow, through the periodic increase of pressure at each pulse. This is analog to what is reported for BFS flow control with plasma actuation, but here with a full 3D geometry due to the annular capillary expansion. In our gas injection geometry, the step is localized $10 \mathrm{~mm}$ upstream of the capillary outlet. According to Figure 16 of the numerical study of Erturk [89], for Re between 600 and 1300, the reattachment point fluctuates roughly between 10 and 30 $\mathrm{X}_{\mathrm{r}} / \mathrm{h}$, depending on the scenario. In our condition, $\mathrm{h}$ is $0.25 \mathrm{~mm}$, which means that $\mathrm{X}_{\mathrm{r}}$ should naturally fluctuate between $2.5 \mathrm{~mm}$ and $7.5 \mathrm{~mm}$, and, thus, be always located inside the capillary over the Re range considered in this work (see Table 2). As so, the flow region expanding downstream of the capillary outlet in quiescent air corresponds to the unsteady readjusting boundary layer (shear layer).

Figure 12 sketches out the vortex pairing mechanism of large-scale coherent vortex rings downstream of the capillary outlet, in the readjusted boundary layer expanding in quiescent air. The evolution of the flow structure, and of the rippled plasma plumes, could be explained by the alternation of vortex rings, through the vortex paring mode. In single HV shot operation, the generation of one vortex pair could be expected. According to Benard et al. [82], the structure is formed during an almost stationary phase (temporal growing without spatial development), and then moved and grown by fluid entrainment. It could correspond to the very beginning of the rippled phase in our condition (see Figure 6(a.) and Table 2), evidenced with double HV shots. Also, the vortex pair generation is triggered by the pressure wave, which propagates at a group velocity higher than the mean flow velocity, according to Table $\mathbf{2}$. The increasing deformation of the flow and of the rippled plasma plumes (see Figure $6(\mathbf{b}$.)) could be due to the growth of the vortex pair while it is entrained by the flow. Thus, the modification of plasma emission intensity, always observed as brighter in the rippled regions could be explained by a local pressure variation. Indeed, one vortex is an attractor to the other, and the pairing generates between those two vortices a local pressure gradient. The brighter plasma emission could be explained by a higher reduced electric field due to local pressure decrease between the vortex pair. Finally, depending on the growth of the vortex pair and on the drop of the argon concentration in the pinched region of the potential core, the IW propagation 
can be completely stopped. It is worth mentioning that Kyle and Sreenivasan [54] have shown that the vortex rings are formed by the intense oscillations in the shear layer, but only when the density ratio between the fluid jet and its surroundings is lower than 0.6. They have described that the pair of vortex rings, once it has grown enough, completely pinches and breaks the jet potential core. They have also indicated that, in this region, the fluid concentration should exhibit a very sharp drop. A radial fluid ejection from the pinched region can also occur (side jet) [54],[90]. It may explain the argon ejection at the left and the right

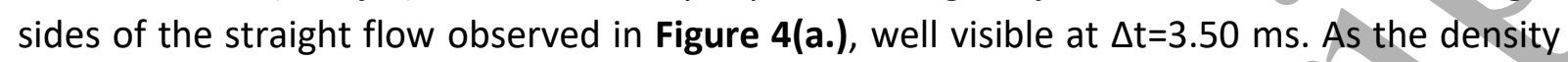
ratio with the argon jet is of about 1.32, we suggest that, in our case, the vortex rings are not initially formed in the argon shear layer downstream of the capillary, but rather inside the capillary (see Figure 11). This may explain why the very beginning of the flow perturbation with the ripple formation cannot be visualized using schlieren imaging, because the argon potential core is pinched by argon vortex rings. However, the very beginning of the flow perturbation can be easily visualized using the double HV shots technique, as the second IW propagates inside the argon potential core. We have also observed that, on the nonaccumulated ICCD images (single acquisition), the bright region in the ripples is present at each pulse, without modification of the filamentary aspect of the IW. This may be an indication that these bright regions in the ripples are not due to air admixture and mainly result from a change of pressure distribution inside the potential core. Nevertheless, time- and spaceresolved spectroscopy is mandatory to evidence or exclude air mixing on the plasma emission, especially as a function of the delays in double HV shots operation. It is likely that air admixture becomes significant during the flow breakdown, and also in the region of the helical arrangement of the plasma plumes when multiple HV shots are used.

When a series of HV pulses are applied, the alternation of the vortex street could change with the PRF, as shown by Benard et al. [82] with plasma actuation. The change of vortex alternation downstream of the capillary outlet could consequently explain the observed change of the ripple alternation in plasma plumes (see Figure 10). According to Yoshioka et al. [91], a forcing at a low PRF allow to promote the formation of large-scale structures with an enhancement of the vortex pairing mechanism. Furthermore, the authors have observed that a forcing at high PRF dissipates the organized motion right after the step, which results in a non-altered flow. This could explain in our case the disappearance of the ripple in the plasma plume and the appearance of a more stable flow structure with a longer straight flow expansion for PRF higher that the transition PRF reported in Figure 9, Table 3 and Table 4. To the extent that the oscillations of the mean reattachment point are dominated by the local forcing due to the HV pulse repetition, the large-scale coherent structures have likely no time to be formed if the oscillations are too fast. This could also explain why the transition PRF for ripple disappearance increases with the flow rate, as the higher is the latter, the higher is the natural frequency of vortex shedding. 


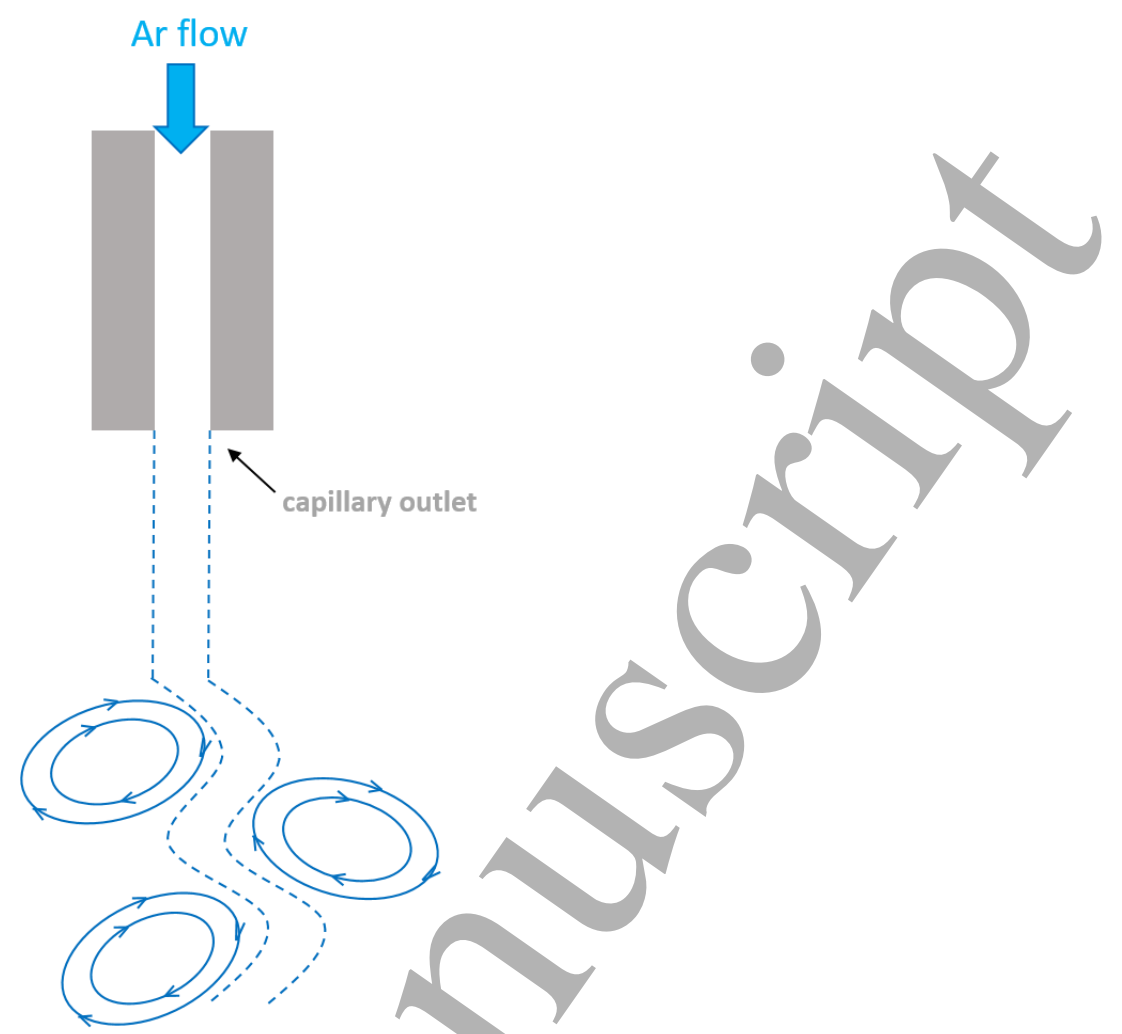

Figure 12 : Schematic $2 D$ view of the vortex pairing mechanism with alternation of large-scale coherent vortex rings downstream of the capillary outlet.

An exhaustive identification and characterization of excited flow modes is beyond the scope of this paper. Nevertheless, we have observed some large coherent structures as those reported in the literature of BFS flow. Figure 13 shows a schlieren image of the argon flow at $300 \mathrm{sccm}$, excited with the repetition of $300 \mathrm{HV}$ pulses at $20 \mathrm{kHz}$. A double helical structure is clearly visible (albeit transiently) inside the flow. It is also visible in Figure 8 in the far downstream region of the flow for a $400 \mathrm{sccm}$ flow rate at $10 \mathrm{kHz}$. The double helical structure in BFS flow has been reported by Sheu et $a l$. [68].

Plasma helical and double helical stationary structure with helium CAPPJ have been previously reported in the literature [61], [92]. We assume that this is also a direct consequence of the forced BFS flow by the periodic plasma perturbations. In [61], the inner holowed HV electrode ( $0.8 \mathrm{~mm}$ exit hole) was inserted in a $4 \mathrm{~mm}$ inner diameter capillary (i.e. expansion ratio of 5). The effect of the PRF on the plasma structure likely relies on the global flow structure reorganization with variations in the large-scale coherent structures. This is also true for the Neon plasma plumes in [53], with variations between helical structure and hairpin like structure, depending on the flow rate-PRF couple. For the chiral plasma (helical shape) reported in [92], the authors are also in the case of a BFS flow geometry, with the use of two connected capillaries of different diameters. 
Multiple HV shots

$300 \mathrm{sccm}$

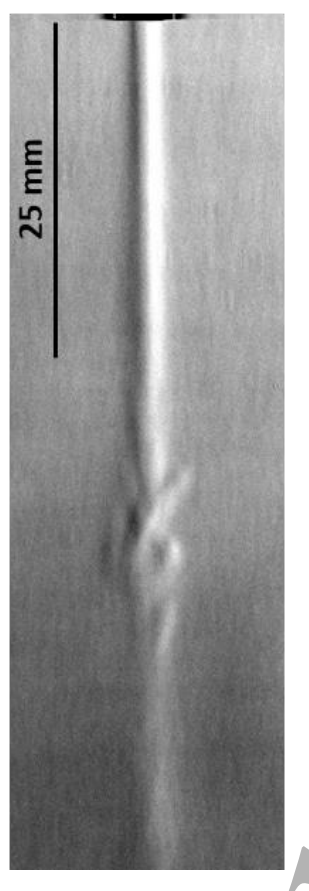

Figure 13 : Schlieren image of the argon flow expansion downstream of the/capillary outlet at $300 \mathrm{sccm}$, excited by the repetition of $300 \mathrm{HV}$ pulses at $20 \mathrm{kHz}(\Delta t=13.9 \mathrm{~ms})$.

Cold atmospheric pressure plasma jet devices could be potentially used as new devices for BFS flow excitation, with the advantage of the natural plasma ignition in the step vicinity, which does not require the modification of the step profile with additional electrode assembly. It also offers a large variety of parameters to investigate; for instance, the electric condition close to the HV electrode is imposed by the position of the grounded electrode. Beyond the variation of the voltage amplitude, the influence of the voltage slope on the flow structure could also be expected [93]. Indeed, the variation of the fast temperature increase rate could change the amplitude of the pressure wave generated at the IW ignition, and, consequently, change the force balance in the reattachment flow region. The major modification of the flow field and instabilities inside the capillary, depending on the gas injection geometry and HV electrode assembly used, could be of importance for the variation of reactive species production from one CAPPJ geometry to another. This reinforces the influence of the electrode configuration on the reactive species production [94].

\section{Conclusions}

In this paper, we have studied the plasma-flow interaction in a cold atmospheric pressure argon plasma jet (CAPPJ) driven by nanosecond HV pulses. A particular attention was paid to the time dynamics of the argon flow structure and plasma plume modifications, resulting from the application of a single HV pulse (single HV shot) to a series of HV pulses at several PRF 
(multiple HV shots). A specifically designed schlieren optical bench with a good sensitivity (in the range of few arcseconds) allowed to visualize the argon flow expansion in quiescent air. Moderate flow rates were investigated, between 400 and $800 \mathrm{sccm}$, chosen for plasma plume expansion in a laminar flow region. It was observed that the application of a single HV pulse is enough to disturb the flow, as previously reported for helium CAPPJ. After the HV pulse application, a ripple propagation was observed in the argon flow, on a timescale related to the flow mean velocity. Using the ability of the IW to follow the denser regions of the argon flow, a double HV shots technique was used, setting the appropriate delay between the two HV shots and integrating the plasma emission over the second HV pulse. Thus, the second IW could be used as a probe, to instantly visualize the flow structure any time after the application of the first HV pulse. For some flow rates, the ripple can increase in amplitude up to the point when it strongly deforms, or even stops, the plasma plume expansion (flow breakdown), after which it is entrained by the flow and the plasma plume retrieves its full usual expansion. The deformation in the rippled region is also systematically associated with a brighter plasma emission. This could result from the pinching of the argon potential core by the pairing of growing vortex rings. Because one vortex ring is an attractor to another, the pairing locally induces a pressure gradient, up to the point where the potential core breaks when the vortex rings have grown enough. The complete pinching of the argon potential core, with the concomitant drop of argon density, likely results in the brutal stopping of the IW propagation. Prior to the flow breakdown, the brighter IW emission could be associated to a local variation of the reduced electric field $\mathrm{E} / \mathrm{N}$, due to the change of pressure distribution inside the pinch of the potential core. In a total non-intrusive way, the propagation of the argon IW inside the argon potential core reflects its topology. Indeed, the IW propagation is not only naturally sensitive to local changes of pressure distribution, but it also directly indicates the end of the argon potential core, by its brutal stop.

Furthermore, when a series of HV pulses were applied and the PRF was progressively increased, precise PRF, specific of each flow rate and increasing with it, were found to maximize the disturbance of the flow (radial splitting). Rippled plasma plumes (3D-helical-like) were observed in the PRF range exactly corresponding to the radial splitting of the flow. Additionally, precise threshold PRF were also found, also specific of each flow rate and also increasing with it, beyond which the flow was clearly less disturbed (straight flow expansion) and straight plasma plumes were observed. We suggest that the formation of coherent vortex structures do not begin in the argon shear layer downstream of the capillary outlet, but rather inside the capillary in the vicinity of the inner hollowed HV electrode. This is a consequence of the gas injection geometry used, because the argon flowing through the HV electrode undergoes a sudden expansion at its outlet, still inside the capillary. This kind of geometry is referenced as backward-facing step (BFS) and has been extensively studied in the field of detached-reattached flow. BFS flows are known for their complex dynamics, with generation of various series of vortices, 3D in nature, through distinct but strongly coupled unstable and unsteady flow regions, over a wide range of periodic modes. Thus, it is likely that the periodic pressure increase in the HV electrode vicinity, at each IW ignition, mechanically forces the flow and locally changes the force balance in the reattachment region. This cause to enhance or inhibit, depending on the forcing frequency with respect to the flow rate, the formation 
and alternation of large coherent vortex structures in the recovered boundary layer region of the BFS flow. This region extends further than the capillary outlet, where the argon flow starts to expand in quiescent air. This plasma effect on the flow structure is analog to what is reported in the literature for flow control by BFS forcing, where plasma actuation effect has been demonstrated. By conception, one advantage of CAPPJ is that the plasma is naturally ignited in the step vicinity and, consequently, it does not require the addition of actuations devices to force the flow. Also, the unique ability of CAPPJ to propagate plasma at long distances from the electrodes could be of interest in the study of forced BFS flow.

The proposed flow control mechanism for CAPPJ only relies on a flow excitation at the appropriate frequencies, through the repetitive pressure increase generated at/each IW ignition. This could be easily generalized to other DC pulsed CAPPJ devices, depending on which instabilities modes are accessible to the flow and to which frequencies the flow instability is sensitive. Because the CAPPJ geometries widely differ from one device to another, the plasma-flow interaction must be studied also taking into account the potential flow instabilities inside the capillary, due to the gas injection geometry and the assembly of the HV electrode. It is worth mentioning that the reduction of the plasma plume length is not always necessarily due to the appearance of turbulence and can occur in a more ordered flow regime, as reported in the present paper.

Acknowledgments: This research work was funded/by the PLASCANCER project (INCaPlanCancer N¹7CP087-00).

\section{Bibliography}

[1] D. B. Graves, "The emerging role of reactive oxygen and nitrogen species in redox biology and some implications for plasma applications to medicine and biology," J. Phys. D. Appl. Phys., vol. 45, ho. 26, p. 263001, 2012, doi: 10.1088/0022-

$3727 / 45 / 26 / 263001$.

[2] X. Lu, G. V Naidis, M. Laroussi, S. Reuter, D. B. Graves, and K. Ostrikov, "Reactive species in non-equilibrium atmospheric-pressure plasmas: Generation, transport, and biological effects," Physics Reports, vol. 630, no. April. pp. 1-84, 2016, doi: 10.1016/j.physrep.2016.03.003.

[3] J. Winter, R. Brandenburg, and K. Weltmann, "Atmospheric pressure plasma jets: an overview of devices and new directions," Plasma Sources Sci. Technol., vol. 24, no. 6, p. 064001, 2015, doi: 10.1088/0963-0252/24/6/064001.

[4] S. Reuter, T. Von Woedtke, and K. D. Weltmann, "The kINPen - A review on physics and chemistry of the atmospheric pressure plasma jet and its applications," J. Phys. D. Appl. Phys., vol. 51, no. 23, 2018, doi: 10.1088/1361-6463/aab3ad.

[5] P. M. Girard et al., "Synergistic Effect of H2O2 and NO2 in Cell Death Induced by Cold 
Atmospheric He Plasma," Sci. Rep., vol. 6, no. 2, pp. 1-17, 2016, doi: 10.1038/srep29098.

[6] T. H. Chung et al., "Cell electropermeabilisation enhancement by non-thermal-plasmatreated PBS," Cancers (Basel)., vol. 12, no. 1, 2020, doi: 10.3390/cancers12010219.

[7] K. Sklias, J. S. Sousa, and P. M. Girard, "Role of short- and long-lived reactive species on the selectivity and anti-cancer action of plasma treatment in vitro," Cancers (Basel)., vol. 13, no. 4, pp. 1-31, 2021, doi: 10.3390/cancers13040615.

[8] K. Fricke, H. Tresp, R. Bussiahn, K. Schröder, T. Von Woedtke, and K. D. Weltmann, "On the use of atmospheric pressure plasma for the bio-decontamination of polymers and its impact on their chemical and morphological surface properties," Plasma Chem. Plasma Process., vol. 32, no. 4, pp. 801-816, 2012, doi: 10.1007/s11090-012-9378-8.

[9] H. Taghvaei, V. S. S. K. Kondeti, and P. J. Bruggeman, “Decomposition of Crystal Violet by an Atmospheric Pressure RF Plasma Jet: The Role of Radicals, Ozone, NearInterfacial Reactions and Convective Transport," Plasma Chem. Plasma Process., vol. 39, no. 4, pp. 729-749, 2019, doi: 10.1007/s11090-019-09965-w.

[10] Z. Machala, B. Tarabová, D. Sersenová, M. Janda, and K. Hensel, “Chemical and antibacterial effects of plasma activated water: Correlation with gaseous and aqueous reactive oxygen and nitrogen species, plasma sources and air flow conditions," J. Phys. D. Appl. Phys., vol. 52, no. 3, 2019, doi: 10.1088/1361-6463/aae807.

[11] X. Damany et al., "Impact of an atmospheric argon plasma jet on a dielectric surface and desorption of organic molecules," Eur. Phys. J. Appl. Phys., vol. 75, 2016, doi: 10.1051/epjap/2016150594.

[12] K. Gazeli et al., "Ar(1s5) absolute radial densities in a ns-pulsed argon plasma jet impinging on dielectric targets at floating potential - plasma action on organic molecules," Plasma Process. Polym., vol. 15, no. 10, 2018, doi: 10.1002/ppap.201800080.

[13] K. Gazeli et al., "Experimental investigation of a ns-pulsed argon plasma jet for the fast desorption of weakly volatile organic compounds deposited on glass substrates at variable electric potential," J. Phys. D. Appl. Phys., vol. 53, no. 47, 2020, doi: 10.1088/1361-6463/aba870.

[14] O. V. Penkov, M. Khadem, W. S. Lim, and D. E. Kim, "A review of recent applications of atmospheric pressure plasma jets for materials processing," J. Coatings Technol. Res., vol. 12, no. 2, pp. 225-235, 2015, doi: 10.1007/s11998-014-9638-z.

[15] F. Fanelli and F. Fracassi, "Atmospheric pressure non-equilibrium plasma jet technology: general features, specificities and applications in surface processing of materials," Surf. Coatings Technol., vol. 322, pp. 174-201, 2017, doi: 10.1016/j.surfcoat.2017.05.027.

[16] K. Gazeli et al., "Effect of the gas flow rate on the spatiotemporal distribution of $\operatorname{Ar}(1 \mathrm{s5})$ absolute densities in a ns pulsed plasma jet impinging on a glass surface," Plasma Sources Sci. Technol., vol. 27, no. 6, 2018, doi: 10.1088/1361-6595/aac5b3.

[17] C. Douat, I. Kacem, N. Sadeghi, G. Bauville, M. Fleury, and V. Puech, "Space-time 
resolved density of helium metastable atoms in a nanosecond pulsed plasma jet: influence of high voltage and pulse frequency," J. Phys. D. Appl. Phys., vol. 49, no. 28, p. 285204, 2016, doi: 10.1088/0022-3727/49/28/285204.

[18] J. Winter, J. S. Sousa, N. Sadeghi, A. Schmidt-Bleker, S. Reuter, and V. Puech, "The spatio-temporal distribution of He metastable atoms in a $\mathrm{MHz}$-driven helium plasma jet is influenced by the oxygen/nitrogen ratio of the surrounding atmosphere," Plasma Sources Sci. Technol., vol. 24, p. 025015, 2015, doi: 10.1088/09630252/24/2/025015.

[19] T. Darny, J. M. Pouvesle, V. Puech, C. Douat, S. Dozias, and E. Robert, "Analysis of conductive target influence in plasma jet experiments through helium metastable and electric field measurements," Plasma Sources Sci. Technol., vol. 26, no. 4, 2017, doi: 10.1088/1361-6595/aa5b15.

[20] E. Es-Sebbar, G. Bauville, M. Fleury, S. Pasquiers, and J. Santos Sousa, "Spatiotemporal distribution of absolute densities of argon metastable 1s 5 state in the diffuse area of an atmospheric pressure nanosecond pulsed argon microplasma jet propagating into ambient air," J. Appl. Phys., vol. 126, no. 7, 2019, doi:10.1063/1.5096407.

[21] S. Hübner, J. S. Sousa, V. Puech, G. M. W. Kroesen, and N. Sadeghi, "Electron properties in an atmospheric helium plasma jet determined by Thomson scattering," J. Phys. D. Appl. Phys., vol. 47, no. 43, p. 432001, 2014, doi: 10.1088/00223727/47/43/432001.

[22] R. Ono, "Optical diagnostics of reactive species in atmospheric-pressure nonthermal plasma," J. Phys. D. Appl. Phys., vol. 49, no. 8, p.083001, 2016, doi: 10.1088/00223727/49/8/083001.

[23] K. Orr, Y. Tang, M. Simeni Simeni, D. Van Den Bekerom, and I. V. Adamovich, "Measurements of electric field in an atmospheric pressure helium plasma jet by the E-FISH method," Plasma Sources Sci. Technol., vol. 29, no. 3, 2020, doi: 10.1088/13616595/ab6e5b.

[24] J. P. Trelles, "Advances and challenges in computational fluid dynamics of atmospheric pressure plasmas," Plasma Sources Sci. Technol., vol. 27, no. 9, 2018, doi: 10.1088/1361-6595/aac9fa.

[25] J. Oh, O. T. Olabanji, C. Hale, R. Mariani, K. Kontis, and J. W. Bradley, "Imaging gas and plasma interactions in the surface-chemical modification of polymers using microplasma jets," Journal of Physics D: Applied Physics, vol. 44, no. 15. p. 155206, 2011, doi: 10.1088/0022-3727/44/15/155206.

[26] Y. Morabit, R. D. Whalley, E. Robert, M. I. Hasan, and J. L. Walsh, "Turbulence and entrainment in an atmospheric pressure dielectric barrier plasma jet," Plasma Process. Polym., vol. 17, no. 6, p. 19000217, 2020, doi: 10.1002/ppap.201900217.

[27] R. D. Whalley and J. L. Walsh, "Turbulent jet flow generated downstream of a low temperature dielectric barrier atmospheric pressure plasma device," Sci. Rep., vol. 6, no. August, pp. 1-7, 2016, doi: 10.1038/srep31756.

[28] E. Robert et al., "Rare gas flow structuration in plasma jet experiments," Plasma 
Sources Sci. Technol., vol. 23, no. 1, p. 012003, 2014, doi: 10.1088/09630252/23/1/012003.

[29] M. Boselli et al., "Schlieren high-speed imaging of a nanosecond pulsed atmospheric pressure non-equilibrium plasma jet," Plasma Chem. Plasma Process., vol. 34, no. 4, pp. 853-869, 2014, doi: 10.1007/s11090-014-9537-1.

[30] P. K. Papadopoulos et al., "Interpretation of the gas flow field modification induced by guided streamer ('plasma bullet') propagation," J. Phys. D. Appl. Phys., vol. 47, no. 42, p. 425203,2014 , doi: 10.1088/0022-3727/47/42/425203.

[31] M. Foletto, V. Puech, J. Fontane, L. Joly, and L. C. Pitchford, "Evidence of the influence of plasma jets on a helium flow into open air," IEEE Trans. Plasma Sci., vol. 42, no. 10, pp. 2436-2437, 2014, doi: 10.1109/TPS.2014.2331393.

[32] M. Ghasemi, P. Olszewski, J. W. Bradley, and J. L. Walsh, "Interaction of multiple plasma plumes in an atmospheric pressure plasma jet array," J. Phys. D. Appl. Phys., vol. 46, no. 5, p. 052001, 2013, doi: 10.1088/0022-3727/46/5/052001.

[33] J. W. Bradley, J. S. Oh, O. T. Olabanji, C. Hale, R. Mariani, and K. Kontis, "Schlieren photography of the outflow from a plasma jet," IEEE Trans. Plasma Sci., vol. 39, no. 11 PART 1, pp. 2312-2313, 2011, doi: 10.1109/TPS.2011.2157940.

[34] N. Jiang, J. Yang, F. He, and Z. Cao, "Interplay of discharge and gas flow in atmospheric pressure plasma jets," J. Appl. Phys., vol. 109, no. 9, 2011, doi: 10.1063/1.3581067.

[35] S. Iseni, a Schmidt-Bleker, J. Winter, K.-D. Weltmann, and S. Reuter, "Atmospheric pressure streamer follows the turbulent argon air boundary in a $\mathrm{MHz}$ argon plasma jet investigated by OH-tracer PLIF spectroscopy," J. Phys. D. Appl. Phys., vol. 47, no. 15, p. 152001, 2014, doi: 10.1088/0022-3727/47/15/152001.

[36] S. Zhang, a Sobota, E. M. van Veldhuizen, and P. J. Bruggeman, "Gas flow characteristics of a time modulated APPJ: the effect of gas heating on flow dynamics," J. Phys. D. Appl. Phys., vol. 48, no. 1, p. 015203, 2015, doi: $10.1088 / 0022-$ $3727 / 48 / 1 / 015203$.

[37] J. Voráč, L. Potočňáková, P. Synek, J. Hnilica, and V. Kudrle, "Gas mixing enhanced by power modulations in atmospheric pressure microwave plasma jet," Plasma Sources Sci. Technol., vol. 25, no. 2, 2016, doi: 10.1088/0963-0252/25/2/025018.

[38] S. Park, U. Cvelbar, W. Choe, and S. Y. Moon, "The creation of electric wind due to the electrohydrodynamic force," Nat. Commun., vol. 9, no. 1, 2018, doi: 10.1038/s41467017-02766-9.

[39] M. Kubečka, M. Snirer, A. Obrusník, V. Kudrle, and Z. Bonaventura, "Computational study of plasma-induced flow instabilities in power modulated atmospheric-pressure microwave plasma jet," Plasma Sources Sci. Technol., vol. 29, no. 7, 2020, doi: 10.1088/1361-6595/ab9b19.

[40] X. Shao, Z. Chang, H. Mu, W.-L. Liao, and G.-J. Zhang, "Experimental and Numerical Investigation on the Interaction Between Ar Flow Channel and Ar Plasma Jet at Atmospheric Pressure," IEEE Trans. Plasma Sci., vol. 41, no. 4, pp. 899-906, 2013. 
[41] I. L. Semenov and K. D. Weltmann, "Modelling of turbulent reacting flow for a cold atmospheric pressure argon plasma jet," Plasma Sources Sci. Technol., vol. 29, no. 5, 2020, doi: 10.1088/1361-6595/ab8273.

[42] D. Passaras, E. Amanatides, and G. Kokkoris, "Predicting the flow of cold plasma jets in kINPen: A critical evaluation of turbulent models," J. Phys. D. Appl. Phys., vol. 53, no. 26, 2020, doi: 10.1088/1361-6463/ab7d6d.

[43] E. R. W. Van Doremaele, V. S. S. K. Kondeti, and P. J. Bruggeman, "Effect of plasma on gas flow and air concentration in the effluent of a pulsed cold atmospheric pressure helium plasma jet," Plasma Sources Sci. Technol., vol. 27, no. 9, 2018, doi:

10.1088/1361-6595/aadbd3.

[44] A. M. Lietz, E. Johnsen, and M. J. Kushner, "Plasma-induced flow instabilities in atmospheric pressure plasma jets," Appl. Phys. Lett., vol. 111, no. 11, 2017, doi: 10.1063/1.4996192.

[45] T. Darny, J. M. Pouvesle, J. Fontane, L. Joly, S. Dozias, and E. Robert, "Plasma action on helium flow in cold atmospheric pressure plasma jet experiments," Plasma Sources Sci. Technol., vol. 26, no. 10, 2017, doi: 10.1088/1361-6595/aa8877.

[46] M. H. Qaisrani, Y. Xian, C. Li, X. Pei, M. Ghasemi, and X. Lu, "Study on dynamics of the influence exerted by plasma on gas flow field in non-thermal atmospheric pressure plasma jet," Phys. Plasmas, vol. 23, no. 6, 2016, doi: 10.1063/1.4954828.

[47] Y. Bin Xian, M. Hasnain Qaisrani, Y. F. Yue, and X. P. Lu, "Discharge effects on gas flow dynamics in a plasma jet," Phys. Plasmas, vol. 23, no. 10, 2016, doi:

10.1063/1.4964784.

[48] M. I. Hasan and J. W. Bradley, "Reassessment of the body forces in a He atmosphericpressure plasma jet: a modelling study," J. Phys. D. Appl. Phys., vol. 49, no. 5, p. 055203, 2016, doi: 10.1088/0022-3727/49/5/055203.

[49] Y. Zheng, L. Wang, W. Ning, and S. Jia, "Schlieren imaging investigation of the hydrodynamics of atmospheric helium plasma jets," J. Appl. Phys., vol. 119, no. 12, 2016, doi: 10.1063/1.4944052.

[50] E. Traldi et al., "Schlieren imaging: a powerful tool for atmospheric plasma diagnostic," EPJ Tech. Instrum., vol. 5, no. 1, pp. 1-23, 2018, doi: 10.1140/epjti/s40485-018-0045-1.

[51] M. Lessen, "On stability of free laminar boundary layer between parallel streams," pp. 571-579, 1949.

[52] A. Michalke, "On spatially growing disturbances in an inviscid shear layer," J. Fluid Mech., vol. 23, no. 3, pp. 521-544, 1965, doi: 10.1017/S0022112065001520.

[53] T. Darny, E. Robert, D. Ries, S. Dozias, and J. M. Pouvesle, "Unexpected plasma plume shapes produced by a microsecond plasma gun discharge," IEEE Trans. Plasma Sci., vol. 42, no. 10, pp. 2504-2505, 2014, doi: 10.1109/TPS.2014.2324893.

[54] D. M. KYLE and K. R. SREENIVASAN, "The instability and breakdown of a round variable-density jet,” J. Fluid Mech., vol. 249, pp. 619-664, 1993. 
[55] B. M. Cetegen, "Behavior of naturally unstable and periodically forced axisymmetric buoyant plumes of helium and helium-air mixtures," Phys. Fluids, vol. 9, no. 12, pp. 3742-3752, 1997, doi: 10.1063/1.869512.

[56] B. M. Cetegen and K. D. Kasper, "Experiments on the oscillatory behavior of buoyant plumes of helium and helium-air mixtures," Phys. Fluids, vol. 8, no. 11, pp. 2974-2984, 1996, doi: 10.1063/1.869075.

[57] G. Michaux and O. Vauquelin, "Density effect on the mixing and the flow pattern of an impinging air-helium jet," Exp. Therm. Fluid Sci., vol. 33, no. 6, pp. 976-982, 2009, doi: 10.1016/j.expthermflusci.2009.04.003.

[58] B. M. B. Cetegen, Y. Dong, and M. C. Soteriou, "Experiments on stability and oscillatory behavior of planar buoyant plumes," Phys. Fluids, vol. 10, no. 7, p. 1658, 1998, doi: 10.1063/1.869683.

[59] K. S. Pasumarthi and A. K. Agrawal, "Schlieren measurements and analysis of concentration field in self-excited helium jets," Phys. Fluids, vol. 15, no. 12, pp. 36833692, 2003, doi: 10.1063/1.1623490.

[60] C. Douat, G. Bauville, M. Fleury, M. Laroussi, and V. Puech, "Dynamics of colliding microplasma jets," Plasma Sources Sci. Technol., vol. 21, no. 3, p. 034010, 2012, doi: 10.1088/0963-0252/21/3/034010.

[61] T. Darny, E. Robert, S. Dozias, and J. Pouvesle, "Helical Plasma Propagation of Microsecond Plasma Gun Discharges," IEEE Trans. Plasma Sci., vol. 42, no. 10, pp. 2506-2507, 2014.

[62] L. Chen, K. Asai, T. Nonomura, G. Xi, and T. Liu, "A review of Backward-Facing Step (BFS) flow mechanisms, heat transfer and control," Therm. Sci. Eng. Prog., vol. 6, no. April, pp. 194-216, 2018, doi: 10.1016/j.tsep.2018.04.004.

[63] G. Biswas, M. Breuer, and F. Durst, "Backward-facing step flows for various expansion ratios at low and moderate reynolds numbers," J. Fluids Eng. Trans. ASME, vol. 126, no. 3, pp. 362-374, 2004, doi: 10.1115/1.1760532.

[64] M. Kiya and K. Sasaki, "Structure of large-scale vortices and unsteady reverse flow in the reattaching zone of a turbulent separation bubble," J. Fluid Mech., vol. 154, pp. 463-491, 1985.

[65] D. Wee, T. Yi, A. Annaswamy, and A. F. Ghoniem, "Self-sustained oscillations and vortex shedding in backward-facing step flows: Simulation and linear instability analysis," Phys. Fluids, vol. 16, no. 9, pp. 3361-3373, 2004, doi: 10.1063/1.1773091.

[66] K. Sasaki and M. Kiya, "Three-dimensional vortex structure in a leading-edge separation bubble at moderate reynolds numbers," J. Fluids Eng. Trans. ASME, vol. 113, no. 3, pp. 405-410, 1991, doi: 10.1115/1.2909510.

[67] H. P. Rani, T. W. H. Sheu, and E. S. F. Tsai, "Eddy structures in a transitional backwardfacing step flow," J. Fluid Mech., vol. 588, no. November 2014, pp. 43-58, 2007, doi: 10.1017/S002211200700763X.

[68] T.W. H. Sheu and H. P. Rani, "Exploration of vortex dynamics for transitional flows in a 
three-dimensional backward-facing step channel," J. Fluid Mech., vol. 550, no. March, pp. 61-83, 2006, doi: 10.1017/S0022112005007858.

[69] T. C. Corke, C. L. Enloe, and S. P. Wilkinson, "Dielectric Barrier Discharge Plasma Actuators for Flow Control*," Annu. Rev. Fluid Mech., vol. 42, no. 1, pp. 505-529, 2010, doi: 10.1146/annurev-fluid-121108-145550.

[70] N. Benard and E. Moreau, "Electrical and mechanical characteristics of surface AC dielectric barrier discharge plasma actuators applied to airflow control," Exp. Fluids vol. 55, no. 11, 2014, doi: 10.1007/s00348-014-1846-x.

[71] K. Kinefuchi, A. Y. Starikovskiy, and R. B. Miles, "Control of shock-wave/boundarylayer interaction using nanosecond-pulsed plasma actuators," J. Propuls. Power, vol. 34, no. 4, pp. 909-919, 2018, doi: 10.2514/1.B36530.

[72] G. S. Settles, Schlieren and shadowgraph techniques: visualizing phenomena in transparent media. New York, 2001.

[73] S. Hübner, S. Hofmann, E. M. van Veldhuizen, and P. J. Bruggeman, "Electron densities and energies of a guided argon streamer in argon and air environments," Plasma Sources Sci. Technol., vol. 22, no. 6, p. 065011, 2013, doi: 10.1088/09630252/22/6/065011.

[74] K. Urabe, Y. Ito, O. Sakai, and K. Tachibana, "Interaction between dielectric barrier discharge and positive streamer in helium plasma jet at atmospheric pressure," Jpn. J. Appl. Phys., vol. 49, no. 10, pp. 1060011-1060016, 2010, doi: 10.1143/JJAP.49.106001.

[75] a Sobota, O. Guaitella, and A. Rousseau, "The influence of the geometry and electrical characteristics on the formation of the atmospheric pressure plasma jet," Plasma Sources Sci. Technol., vol. 23, no. 2, p. 025016, 2014, doi: 10.1088/09630252/23/2/025016.

[76] O. Gazeli, C. Lazarou, G. Niu, C. Anastassiou, G. E. Georghiou, and J. Franzke, "Propagation dynamics of a helium micro-tube plasma: Experiments and numerical modeling," Spectrochim. Acta-Part B At. Spectrosc., vol. 182, no. February, p. 106248, 2021, doi: 10.1016/j.sab.2021.106248.

[77] J. Jánský and A. Bourdon, "Surface charge deposition inside a capillary glass tube by an atmospheric pressure discharge in air," EPJ Appl. Phys., vol. 55, no. 1, pp. 1-8, 2011, doi: 10.1051/epjap/2011100504.

[78] J. Jansky, P. Le Delliou, F. Tholin, P. Tardiveau, A. Bourdon, and S. Pasquiers, "Experimental and numerical study of the propagation of a discharge in a capillary tube in air at atmospheric pressure," J. Phys. D. Appl. Phys., vol. 44, no. 33, 2011, doi: 10.1088/0022-3727/44/33/335201.

[79] J. K. Eaton and J. P. Johnston, "A review of research on subsonic turbulent flow reattachment," AIAA J., vol. 19, no. 9, pp. 1093-1100, 1981, doi: 10.2514/3.60048.

[80] T. R. Troutt, B. Scheelke, and T. R. Norman, "Organized structures in a reattaching separated flow field," J. Fluid Mech., vol. 143, pp. 413-427, 1984, doi: $10.1017 /$ S0022112084001415. 
[81] L. W. Sigurdson, "The structure and control of a turbulent reattaching flow," J. Fluid Mech., vol. 298, pp. 139-165, 1995, doi: 10.1017/S0022112095003259.

[82] N. Benard, P. Sujar-Garrido, J. P. Bonnet, and E. Moreau, "Control of the coherent structure dynamics downstream of a backward facing step by DBD plasma actuator," Int. J. Heat Fluid Flow, vol. 61, pp. 158-173, 2016, doi: 10.1016/j.ijheatfluidflow.2016.04.009.

[83] E. Stephen, N. Carter, C. Leung, C. Sumerel, C. Y. Hsu, and T. McLaughlin, "Flow overa backward-facing step under controlled-plasma actuator-induced perturbations," 40th AIAA Fluid Dyn. Conf., no. July, pp. 1-13, 2010, doi: 10.2514/6.2010-4591.

[84] J. D'Adamo, R. Sosa, and G. Artana, "Active control of a backward facing step flow with plasma actuators," J. Fluids Eng. Trans. ASME, vol. 136, no. 12, pp. 1-9, 2014, doi: 10.1115/1.4027598.

[85] P. Sujar-Garrido, N. Benard, E. Moreau, and J. P. Bonnet, "Dielectric barrier discharge plasma actuator to control turbulent flow downstream of a backward-facing step," Exp. Fluids, vol. 56, no. 4, 2015, doi: 10.1007/s00348-015-1939-1.

[86] Y. Koide, R. Sasaki, Y. Kameya, and M. Motosuke, "A burst wave-induced plasma actuator for controlling separated flow over a backward-facing step at low Reynolds numbers," Exp. Therm. Fluid Sci., vol. 66, pp. 72-78, 2015, doi:

10.1016/j.expthermflusci.2015.03.016.

[87] S. G. Pouryoussefi, M. Mirzaei, and M. Hajipour, "Experimental study of separation bubble control behind a backward-facing step using plasma actuators," Acta Mech., vol. 226, no. 4, pp. 1153-1165, 2015, doi: 10.1007/s00707-014-1245-7.

[88] R. Ruisi, H. Zare-Behtash, K. Kontis, and R. Erfani, "Active flow control over a backward-facing step using plasma actuation," Acta Astronaut., vol. 126, pp. 354-363, 2016, doi: 10.1016/j.actaastro.2016.05.016.

[89] E. Erturk, "Numerical solutions of 2-D steady incompressible flow over a backwardfacing step, Part I: High Reynolds number solutions," Comput. Fluids, vol. 37, no. 6, pp. 633-655, 2008, doi: 10.1016/j.compfluid.2007.09.003.

[90] P. A. Monkewitz, B. Lehmann, B. Barsikow, and D. W. Bechert, "The spreading of selfexcited hot jets by side jets," Phys. Fluids A, vol. 1, no. 3, pp. 446-448, 1989, doi: $10.1063 / 1.857467$.

[91] S. Yoshioka, S. Obi, and S. Masuda, "Organized vortex motion in periodically perturbed turbulent separated flow over a backward-facing step," Int. J. Heat Fluid Flow, vol. 22, no. 3, pp. 301-307, 2001, doi: 10.1016/S0142-727X(01)00092-3.

[92] S. Jin, D. Zou, X. Lu, and M. Laroussi, "The effect of tube geometry on the chiral plasma," Phys. Plasmas, vol. 26, no. 9, 2019, doi: 10.1063/1.5120288.

[93] Y. Zhu, Y. Wu, M. Jia, H. Liang, J. Li, and Y. Li, "Influence of positive slopes on ultrafast heating in an atmospheric nanosecond-pulsed plasma synthetic jet," Plasma Sources Sci. Technol., vol. 24, p. 015007, 2015, doi: 10.1088/0963-0252/24/1/015007.

[94] A. M. Lietz and M. J. Kushner, "Electrode configurations in atmospheric pressure 
plasma jets: Production of reactive species," Plasma Sources Sci. Technol., vol. 27, no. 10, 2018, doi: 10.1088/1361-6595/aadf5b. 